University of Nebraska - Lincoln

DigitalCommons@University of Nebraska - Lincoln

September 1973

\title{
Magnetic Interactions and High-Field Magnetization in Dilute Magnetic Alloys
}

J.M. Franz

Massachusetts Institute of Technology, Cambridge, Massachusetts

David J. Sellmyer

University of Nebraska-Lincoln, dsellmyer@unl.edu

Follow this and additional works at: https://digitalcommons.unl.edu/physicssellmyer

Part of the Physics Commons

Franz, J.M. and Sellmyer, David J., "Magnetic Interactions and High-Field Magnetization in Dilute Magnetic Alloys " (1973). David Sellmyer Publications. 178.

https://digitalcommons.unl.edu/physicssellmyer/178

This Article is brought to you for free and open access by the Research Papers in Physics and Astronomy at DigitalCommons@University of Nebraska - Lincoln. It has been accepted for inclusion in David Sellmyer Publications by an authorized administrator of DigitalCommons@University of Nebraska - Lincoln. 


\title{
Magnetic Interactions and High-Field Magnetization in Dilute Magnetic Alloys*
}

\author{
J. M. Franz ${ }^{\dagger}$ \\ Massachusetts Institute of Technology, Cambridge, Massachusetts 02139 \\ D. J. Sellmyer \\ Massachusetts Institute of Technology, Cambridge, Massachusetts 02139 \\ Behlen Laboratory of Physics, $¥$ University of Nebraska, Lincoln, Nebraska 68508
}

(Received 9 March 1973)

\begin{abstract}
Magnetic interactions have been investigated in $\mathrm{Cu}(\mathrm{Mn})$ and $\mathrm{Cu}(\mathrm{Fe})$ alloys with concentrations ranging from several hundred to several thousand ppm. In each system two sets of alloys were prepared in different ways to test for sensitivity to structrual modifications. In the $\mathrm{Cu}(\mathrm{Mn})$ alloys there was very little difference in magnetic properties of the two sets of alloys which is consistent with the expectation that the $\mathrm{Cu}(\mathrm{Mn})$ alloys form good random solid solutions. The magnetic properties were compared with the recent mean-random-field theory of Klein in the low-temperature limit. It was found that the theory is able to explain the concentration dependence of the initial susceptibility and also gives a semiquantitatively correct description of the magnetization to $100 \mathrm{kOe}$. In the $\mathrm{Cu}(\mathrm{Fe})$ alloys the results confirm in part recent work of Tholence and Tournier who, working with much more dilute samples, showed that there exist isolated $\mathrm{Fe}$ atoms and ferromagnetically coupled $\mathrm{Fe}-\mathrm{Fe}$ pairs. The pair magnetization saturates at $\sim 60 \mathrm{kOe}$ for $T \lesssim 4^{\circ} \mathrm{K}$ and each pair has associated with it a spin of $\simeq 3$. The pair concentration was determined from magnetization and recent Mössbauer experiments in several different sets of alloys. It was shown that this pair density depends sensitively on sample-preparation techniques and cold work, i.e., on modifications of the structure of these supersaturated alloys. It is therefore argued that the pairs are not coupled by long-range Ruderman-Kittel-type interactions but rather they should be regarded as near-neighbor pairs acting as a diatomic molecule dissolved in the copper matrix. The most concentrated alloys in each set of samples $(0.6$ at.\%) exhibited remanence below $3.5^{\circ} \mathrm{K}$. The magnitude of the remanence was proportional to the pair densities in the respective sample sets, suggesting that the ordering was a cooperative effect between the pairs through a mechanism that is not clearly defined. It was shown how the spin-glass transitions recently observed by Svensson in cold-worked alloys could be understood in terms of the breaking up of pairs and other clusters-with a subsequent return to a more random alloy. Finally, the $1.6^{\circ} \mathrm{K}$ spin polarization associated with an isolated $\mathrm{Fe}$ impurity to $\approx 100 \mathrm{kOe}$ is compared to two Brillouin functions; one corresponding to $T=1.6^{\circ} \mathrm{K}$ and the second to $T=30.6^{\circ} \mathrm{K}\left(\simeq 1.6+T_{K}\right)$. The second function fits the data fairly well but there is no theoretical justification for this agreement.
\end{abstract}

\section{INTRODUCTION}

The understanding of magnetism in metals involves two fundamental concepts. The first of these concerns the formation of a magnetic moment on the atom in the presence of the conduction electrons of the metal. The second involves the long-range interactions between moments, which lead to ferromagnetism or antifer romagnetism. By studying the properties of small amounts of magnetic impurity in a nonmagnetic host, one might hope to eliminate the impurity-impurity interactions and concentrate on the properties of the single impurity. Theoretical work by Friedel and by Anderson ${ }^{1}$ has led to an understanding of the conditions under which moments form on impurity atoms in metals. By considering the scattering of conduction electrons by well-defined moments on impurities, Kondo ${ }^{2}$ explained the resistance minimum observed in these alloys. This led to the present understanding of the single-impurity problem (Kondo effect), which is extensively reviewed theoretically and experimentally by Kondo ${ }^{3}$ and Heeger. ${ }^{4}$
In the single-impurity limit it is found that an alloy may be characterized by a parameter $T_{K}$, the Kondo temperature, which is independent of impurity concentration. As impurity concentration is increased, interactions between moments via the conduction electrons become important and it is these interactions that are of interest in the present work.

Both $\mathrm{Cu}(\mathrm{Fe})$ and $\mathrm{Cu}(\mathrm{Mn})$ have been studied ${ }^{4}$ in the dilute limit with $\mathrm{Cu}(\mathrm{Fe})$ being a classical example of a Kondo system with $T_{K} \simeq 10^{\circ} \mathrm{K} .{ }^{4} \mathrm{Cu}(\mathrm{Mn})$, on the other hand, has $T_{K} \simeq 0.002{ }^{\circ} \mathrm{K}^{5}$ and exhibits resistivity and susceptibility maxima at higher concentrations. It is expected that the magnetic properties of the two systems in the concentration range where impurity-impurity interactions become important, will reflect these differences.

The purpose of this work was to study the magnetic properties of both $\mathrm{Cu}(\mathrm{Mn})$ and $\mathrm{Cu}(\mathrm{Fe})$ over a range of concentrations extending from the region dominated by single-impurity effects to a region dominated by impurity-impurity interaction effects. The magnetic properties of $\mathrm{Cu}(\mathrm{Mn})$ have been 
studied ${ }^{5-9}$ over a wide range of concentrations and temperatures, with most effort on low-field susceptibility in the concentration range where interaction effects dominate the magnetic properties. On the other hand, the magnetic properties of $\mathrm{Cu}(\mathrm{Fe})$ have been studied ${ }^{4,10-13}$ with emphasis on the single-impurity problem. Recent studies of $\mathrm{Cu}(\mathrm{Fe})$ by Tholence and Tournier ${ }^{13}$ indicate that interactions may exist for impurity concentrations below $300 \mathrm{ppm}$, a concentration region previously expected to be in the single-impurity limit. ${ }^{14}$ Thus studies involving interactions have shed new light on the "single"-impurity problem in $\mathrm{Cu}(\mathrm{Fe})$.

In the present investigation the approach was to obtain more information on interacting moments by studying the way they magnetize in high magnetic fields. To do this we have measured the magnetization of the alloys in magnetic fields to approximately $100 \mathrm{kOe}$ at liquid-helium temperatures, and the initial susceptibility to temperatures of approximately $50^{\circ} \mathrm{K}$.

Recent calculations by Klein ${ }^{15}$ give expressions for low-temperature initial susceptibility and fielddependent magnetization for interacting moments. Attempts to apply this theory to the magnetization of $\mathrm{Cu}(\mathrm{Fe})$ and $\mathrm{Cu}(\mathrm{Mn})$ alloys will be discussed, and a comment will be made on the high-field magnetization of a single spin for temperatures much below the Kondo temperature. Preliminary accounts of this work have been given elsewhere. ${ }^{16}$

\section{EXPERIMENTAL PROCEDURE}

\section{A. Sample Preparation}

$\mathrm{Cu}(\mathrm{Fe})$ and $\mathrm{Cu}(\mathrm{Mn})$ alloys were prepared using 99. $9999 \% \mathrm{Cu}$ (supplied by Cominco American, Inc.). The solute metals were Ferrovac-E Fe of approximately $99.99 \%$ (supplied by Crucible Steel Co. of America) and $99.9 \% \mathrm{Mn}$ (supplied by Johnson Matthey). As explained more fully below, two sets of each alloy system were prepared in order to test the magnetic properties for sensitivity to structural differences produced by varying samplepreparation procedures. This is particularly important for the $\mathrm{Cu}(\mathrm{Fe})$ alloys, which are supersaturated, and we shall return to considerations of this nature in the following sections. The constituents were melted in zirconium oxide crucibles in a vacuum induction furnace and held approximately $100{ }^{\circ} \mathrm{C}$ above the melting temperature $\mathrm{e}^{17}$ for $1 \mathrm{~h}$. The melt was chill cast under positive pressure of helium into a split copper mold cooled with liquid nitrogen. All samples were made from master alloys of approximately 1-at. \% solute content. Two samples in the shape of cylinders $(0.190 \times 0.190$ in.) were machined from the ingot of about 0.250in. diameter. The cylinders were heavily chemically etched, and one was annealed. $\mathrm{Cu}(\mathrm{Mn})$ sam-
TABLE I. Analyzed sample concentration.

\begin{tabular}{rrr}
\hline \hline & \multicolumn{2}{c}{ Impurity concentration } \\
Sample & $(\mathrm{ppm})$ & at. \% \\
\hline $\mathrm{Cu}(\mathrm{Fe})-1$ & 447 & $4.47 \times 10^{-2}$ \\
-2 & 842 & $8.42 \times 10^{-2}$ \\
-3 & 1370 & $1.37 \times 10^{-1}$ \\
-4 & 1750 & $1.75 \times 10^{-1}$ \\
-5 & 2300 & $2.30 \times 10^{-1}$ \\
-6 & 6140 & $6.14 \times 10^{-1}$ \\
$\mathrm{Cu}(\mathrm{Fe})-1 A$ & 447 & $4.47 \times 10^{-2}$ \\
$-2 A$ & 842 & $8.42 \times 10^{-2}$ \\
$-3 A$ & 1270 & $1.27 \times 10^{-1}$ \\
$-4 A$ & 1750 & $1.75 \times 10^{-1}$ \\
$-5 A$ & 2410 & $2.41 \times 10^{-1}$ \\
$-6 A$ & 5960 & $5.96 \times 10^{-1}$ \\
$\mathrm{Cu}(\mathrm{Mn})-2 A$ & 430 & $4.30 \times 10^{-2}$ \\
$-3 A$ & 848 & $8.48 \times 10^{-2}$ \\
$-4 A$ & 1310 & $1.31 \times 10^{-1}$ \\
$-5 A$ & 3430 & $3.43 \times 10^{-1}$ \\
\hline \hline
\end{tabular}

ples were annealed in sealed Vycor tubes evacuated to $1 \times 10^{-6}$ Torr for $40 \mathrm{~h}$ at $500{ }^{\circ} \mathrm{C}$ and allowed to oven cool to room temperature. $\mathrm{Cu}(\mathrm{Fe})$ samples were sealed in Vycor tubes containing high-purity argon (less than 1-ppm $\mathrm{O}_{2}$ ) and held at $900{ }^{\circ} \mathrm{C}$ for $48 \mathrm{~h}$ before being quenched rapidly in ice water in a process that broke the Vycor tube. Impurity concentrations $c$ determined by atomic-absorption spectroscopy are given in Table I. Although it is difficult to determine the absolute accuracy of this analysis, it is estimated to be $\pm 3.0 \%$. Low-field measurements of $\mathrm{Cu}(\mathrm{Mn})$ alloys showed no measurable differences between annealed and unannealed samples. In order to improve the accuracy of the impurity concentrations, the unnealed $\mathrm{Cu}(\mathrm{Mn})$ samples were actually used (i.e., dissolved) for a spectroscopic analysis and these samples are not listed in Table I.

\section{B. Measurement Techniques}

A Princeton Applied Research vibrating-sample magnetometer was used to measure bulk magnetization of the $\mathrm{Cu}(\mathrm{Fe})$ and $\mathrm{Cu}(\mathrm{Mn})$ alloys. Magnetization measurements were made between 1.6 and approximately $50^{\circ} \mathrm{K}$ in fields up to $10 \mathrm{kOe}$ using a conventional electromagnet.

High-field measurements to $95 \mathrm{kOe}$ at 1.6 and 4. $4^{\circ} \mathrm{K}$ were made using an $\mathrm{RCA} \mathrm{Nb}_{3} \mathrm{Sn}$ superconducting solenoid. A Janis Vari-Temp Dewar was employed in both magnets. Temperature was regulated electronically for low-field measurements above $4.2^{\circ} \mathrm{K}$. Regulated temperatures below 4. $2{ }^{\circ} \mathrm{K}$ were achieved by pumping-with a Walker regulator-on liquid helium introduced into the sample chamber. Sample temperature was determined using a calibrated GaAs diode (Lake Shore Cryo- 
tronics, Inc.) periodically introduced into the sample area. It is estimated that the temperature is known to about $\pm 3.0 \%$ over the entire range. The magnetic field is known to approximately $\pm 1.5 \%$ for the low-field measurement, and to $\pm 2.0 \%$ for high-field data. The magnitude of the magnetization was calibrated with respect to the moment of pure nickel ${ }^{18}\left(57.5 \mathrm{emu}\right.$ at $\left.4.4^{\circ} \mathrm{K}\right)$. Details of the apparatus, temperature control, and calibration are given elesewhere. ${ }^{16}$ All measurements of alloy magnetization were corrected for the diamagnetism of pure copper given by $\operatorname{Hurd}^{19}$ as $\chi_{\mathrm{Cu}}$ $=-88.11 \times 10^{-9} \mathrm{emu} / \mathrm{g}$. Initial susceptibility is defined as $\chi_{i}=(d M / d H)_{H \leq 500 O_{e}}$ and high-field susceptibility is given by $\chi_{\mathrm{HF}}=(d M / d H)_{\mathrm{HF}}$, where $M$ is the impurity magnetization and $H$ is the magnetic field.

Most data for each sample were taken several times to determine repeatability. Estimates of the uncertainties in magnetization and susceptibility measurements are shown by error bars on the figures.

\section{EXPERIMENTAL RESULTS}

\section{A. Low-field Susceptibility}

Figure 1 is a plot of inverse susceptibility versus $T$ for the set of $\mathrm{Cu}(\mathrm{Mn})$ alloys. For temperatures above $10^{\circ} \mathrm{K}$ all samples show approximately linear behavior indicative of a Curie-Weiss (C-W) susceptibility. Although a $\mathrm{C}-\mathrm{W}$ analysis is not rigorously applicable, ${ }^{20}$ it serves as a comparison to measurements on more dilute noninteracting moments. The $\mathrm{C}-\mathrm{W}$ law gives

$$
\chi_{i}=\left(n \mu_{\text {eff }}^{2} / 3 k_{B}\right)(T-\theta)^{-1} \text {, }
$$

where $\chi_{i}$ is the initial impurity susceptibility per unit mass, $n$ is the number of impurity atoms per unit mass of alloy, $\theta$ is the Weiss temperature, $\mu_{\text {eff }}$ is the effective moment per impurity, and the other symbols have their usual meanings. If we take $\mu_{\text {eff }}=g[S(S+1)]^{1 / 2} \mu_{B}$, where $\mu_{B}$ is the Bohr magneton, $g=2.0$, and $S$ is the spin on an impurity,

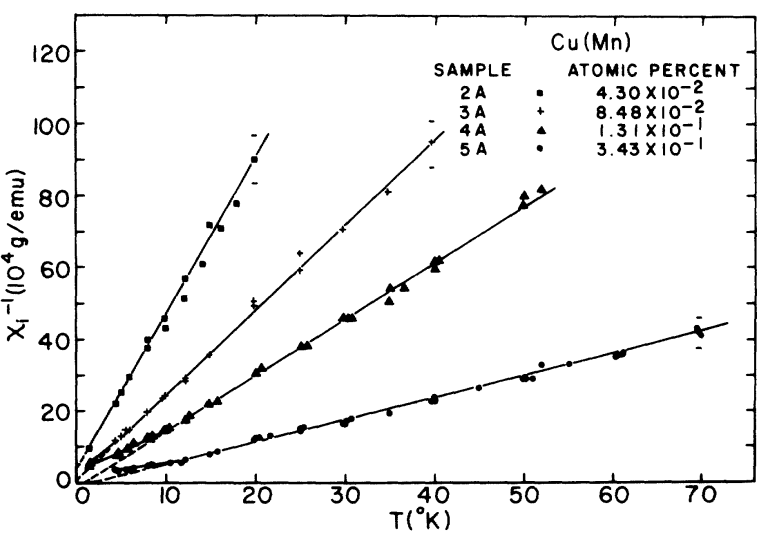

FIG. 1. Temperature dependence of inverse susceptibility for $\mathrm{Cu}(\mathrm{Mn})$ alloys.

we may calculate $\theta, \mu_{\text {esf }}$, and $S$. The results of this calculation are given in Table II. These values can be compared to measurements by $\mathrm{Hurd}^{8}$ on $\mathrm{Cu}(\mathrm{Mn})$ for lower concentrations $(c<200 \mathrm{ppm})$, which give $\mu_{\text {eff }}=(4.93 \pm 0.025) \mu_{B}$ and an average $\theta$ of $(-14 \pm 3)^{\circ} \mathrm{K}$. More recent measurements ${ }^{5}$ at very low temperatures $\left(T \sim 200 \mathrm{~m}^{\circ} \mathrm{K}\right)$ and concentrations $(c<100 \mathrm{ppm})$ give a concentration-independent $\theta \simeq 10 \mathrm{~m}^{\circ} \mathrm{K}$ and $\mu_{\text {eff }}=(4.5 \pm 0.4) \mu_{B}$. Measurements by others ${ }^{6}$ in concentration regions above 0.02 at. \% generally indicate that $\theta$ moves toward positive values with increasing impurity concentration. For temperatures below $10^{\circ} \mathrm{K}$, deviations from the $\mathrm{C}-\mathrm{W}$ law are associated with the apparent onset of antifer romagnetism observed in $\mathrm{Cu}(\mathrm{Mn}) .{ }^{6,7}$ In Fig. 2 the smoothed data of Fig. 1 are normalized using sample concentration. This plot indicates clearly that $\chi_{i}$ above $10^{\circ} \mathrm{K}$ is approximately proportional to $c$ and that significant deviations are observed for $T=1.6{ }^{\circ} \mathrm{K}$.

A plot of $c / \chi_{i}$ vs $T$ for the unannealed set of $\mathrm{Cu}(\mathrm{Fe})$ alloys is given in $\mathrm{Fig} .3$ and indicates that $\chi_{i}$ is not simply proportional to $c$. The down turn of $c / \chi_{i}$ at low temperatures observed for samples $1-5$ has been recently observed ${ }^{13}$ for this system

TABLE II. Curie-Weiss analysis for $\mathrm{Cu}(\mathrm{Mn})$ and $\mathrm{Cu}(\mathrm{Fe})$ alloys.

\begin{tabular}{rcrrr}
\hline \multicolumn{1}{c}{ Sample } & Concentration (at. \%) & \multicolumn{1}{c}{$\mu_{\text {eff }} / \mu_{B}$} & $\theta\left({ }^{\circ} \mathrm{K}\right)$ & \multicolumn{1}{c}{$s$} \\
\hline $\mathrm{Cu}(\mathrm{Mn})-2 A$ & $4.30 \times 10^{-2}$ & $5.2 \pm 0.5$ & $-0.8 \pm 0.5$ & $2.1 \pm 0.2$ \\
$-3 A$ & $8.48 \times 10^{-2}$ & $5.0 \pm 0.5$ & $0.0 \pm 0.5$ & $2.0 \pm 0.2$ \\
$-4 A$ & $1.31 \times 10^{-1}$ & $5.0 \pm 0.5$ & $+1.0 \pm 0.5$ & $2.0 \pm 0.2$ \\
$-5 A$ & $3.43 \times 10^{-1}$ & $4.9 \pm 0.5$ & $+2.0 \pm 0.5$ & $2.0 \pm 0.2$ \\
$\mathrm{Cu}(\mathrm{Fe})-1$ & $4.47 \times 10^{-2}$ & $3.3 \pm 0.3$ & $-10.4 \pm 0.5$ & $1.2 \pm 0.2$ \\
-2 & $8.42 \times 10^{-2}$ & $3.3 \pm 0.3$ & $-6.8 \pm 0.5$ & $1.2 \pm 0.2$ \\
-3 & $1.37 \times 10^{-1}$ & $3.5 \pm 0.3$ & $-4.6 \pm 0.5$ & $1.3 \pm 0.2$ \\
-4 & $1.75 \times 10^{-1}$ & $3.9 \pm 0.4$ & $-3.6 \pm 0.5$ & $1.5 \pm 0.2$ \\
-5 & $2.30 \times 10^{-1}$ & $4.0 \pm 0.4$ & $-1.6 \pm 0.5$ & $1.6 \pm 0.2$ \\
-6 & $6.14 \times 10^{-1}$ & $5.9 \pm 0.5$ & $+0.6 \pm 0.5$ & $2.5 \pm 0.2$ \\
\hline \hline
\end{tabular}




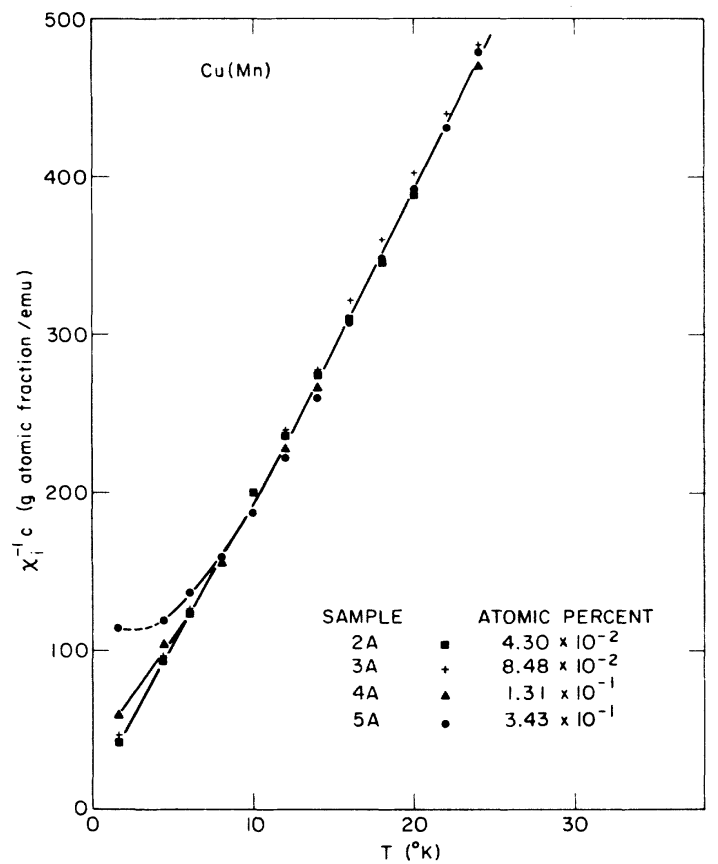

FIG. 2. $c / \chi_{i}$ vs $T$ for $\mathrm{Cu}(\mathrm{Mn})$ alloys.

at concentrations below $600 \mathrm{ppm}$ and is associated with a susceptibility containing both $c$ and $c^{2}$ dependences. The change in character of $c / \chi_{i}$, from turning down at low $T$ to nearly turning up (i.e., flattening) for $\mathrm{Cu}(\mathrm{Fe})-6$, will be discussed in Sec. V. Similar data for annealed $\mathrm{Cu}(\mathrm{Fe})$ samples show the same characteristics while being less magnetic. Table II is given as a comparison with the $\mathrm{Cu}(\mathrm{Fe})$ data of Hurd ${ }^{11}$ which give $\mu_{\text {eff }}=(3.68$ $\pm 0.20) \mu_{B}, \theta=(-32 \pm 2)^{\circ} \mathrm{K}$, and $S=1.4$, for the single-impurity values.

\section{B. Remanence}

At $T=1.6{ }^{\circ} \mathrm{K}, \mathrm{Cu}(\mathrm{Mn})-5$ and $\mathrm{Cu}(\mathrm{Fe})-6$ and $-6 A$ showed remanent magnetization as indicated in Figs. 4(a) and 4(b). These data were taken by cooling the samples in zero field to $1.6{ }^{\circ} \mathrm{K}$, taking data from the origin of Figs. 4(a) and 4(b) to 1.0 kOe or $300 \mathrm{Oe}$, respectively, increasing the field to $10 \mathrm{kOe}$ and returning it to $1.0 \mathrm{kOe}$ or $300 \mathrm{Oe}$, taking data again as the field was decreased to zero and then increased in the opposite direction, etc. At $1.6^{\circ} \mathrm{K}$ the remanent magnetization for the annealed sample $[\mathrm{Cu}(\mathrm{Fe})-6 A]$ was smaller than for the unannealed one $[\mathrm{Cu}(\mathrm{Fe})-6]: M_{r}^{A}=81 \times 10^{-4}$ $\mathrm{emu} / \mathrm{g}$, whereas $M_{r}=105 \times 10^{-4} \mathrm{emu} / \mathrm{g}$. For both $\mathrm{Cu}(\mathrm{Fe})$ alloys, the remanence disappeared for $T$ $>(3.5 \pm 0.2)^{\circ} \mathrm{K}$. For $\mathrm{Cu}(\mathrm{Mn})-5$, remanence was absent at $T=4.4^{\circ} \mathrm{K}$, though no measurements were made between 1.6 and $4.4^{\circ} \mathrm{K}$ to determine an ordering temperature.

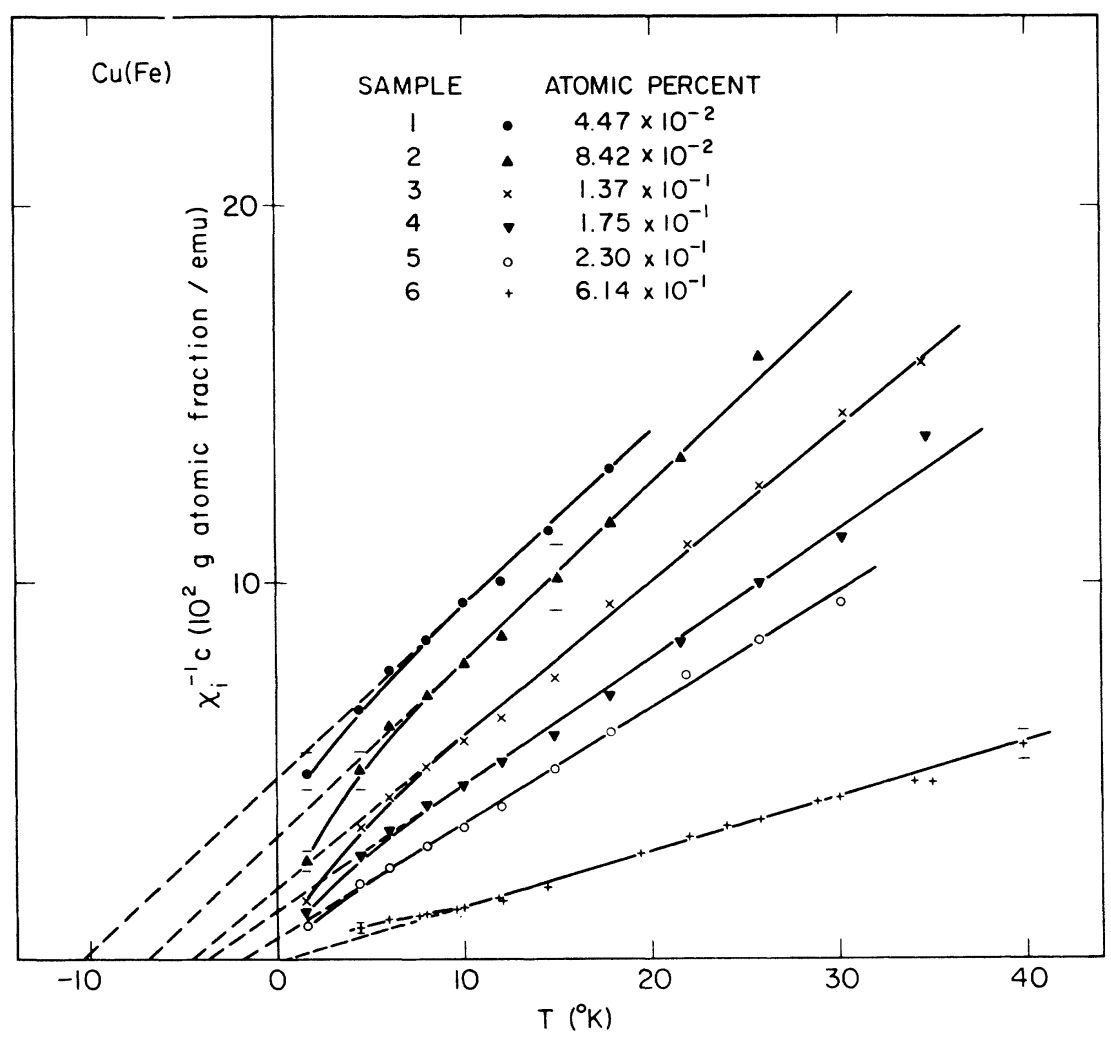

FIG. 3. $c / \chi_{i}$ vs $T$ for $\mathrm{Cu}(\mathrm{Fe})$ alloys (unannealed samples). 

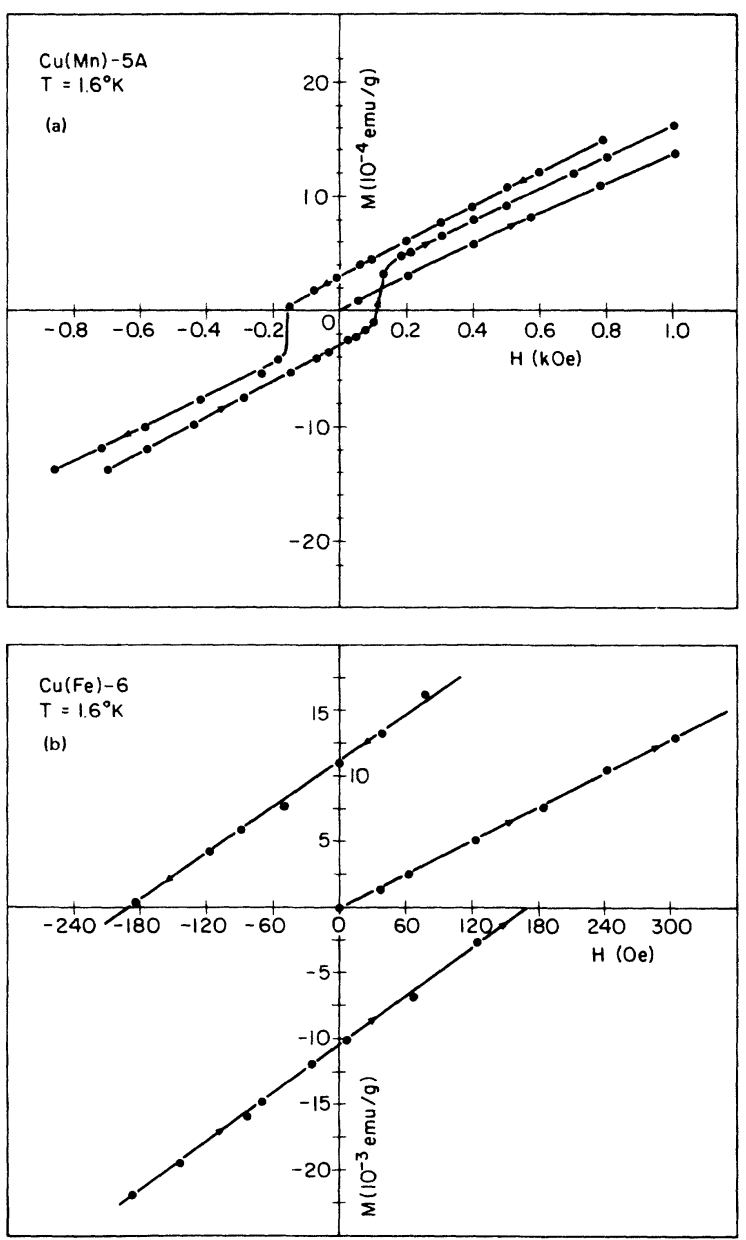

FIG. 4. Remanent magnetization at $T=1.6^{\circ} \mathrm{K}$ for (a) $\mathrm{Cu}(\mathrm{Mn})-5 \mathrm{~A}$ and $(\mathrm{b}) \mathrm{Cu}(\mathrm{Fe})-6$ (unannealed sample). Samples cooled in zero field.

\section{High-Field Magnetization}

High-field measurements to $95 \mathrm{kOe}$ at 1.6 and 4. $4{ }^{\circ} \mathrm{K}$ were made for all unannealed $\mathrm{Cu}(\mathrm{Fe})$ samples and at $T=1.6{ }^{\circ} \mathrm{K}$ for the annealed set. The results for the unannealed samples at $1.6{ }^{\circ} \mathrm{K}$ are shown in Fig. 5. Subsequent analysis has indicated uncertainty in the data at $1.6{ }^{\circ} \mathrm{K}$ for $\mathrm{Cu}(\mathrm{Fe})-1$, and it has been deleted from Fig. 5. Similar measurements for the annealed $\mathrm{Cu}(\mathrm{Mn})$ alloys are shown for $T=1.6{ }^{\circ} \mathrm{K}$ in $\mathrm{Fig}$. 6 .

\section{THEORETICAL CONCEPTS}

\section{A. Random Molecular-Field Model}

The method of random molecular fields has been used with some success to describe the magnetic $^{15,21,22}$ and transport ${ }^{23}$ properties of certain alloys, sometimes called "spin glasses," containing dilute concentrations of interacting magnetic im-

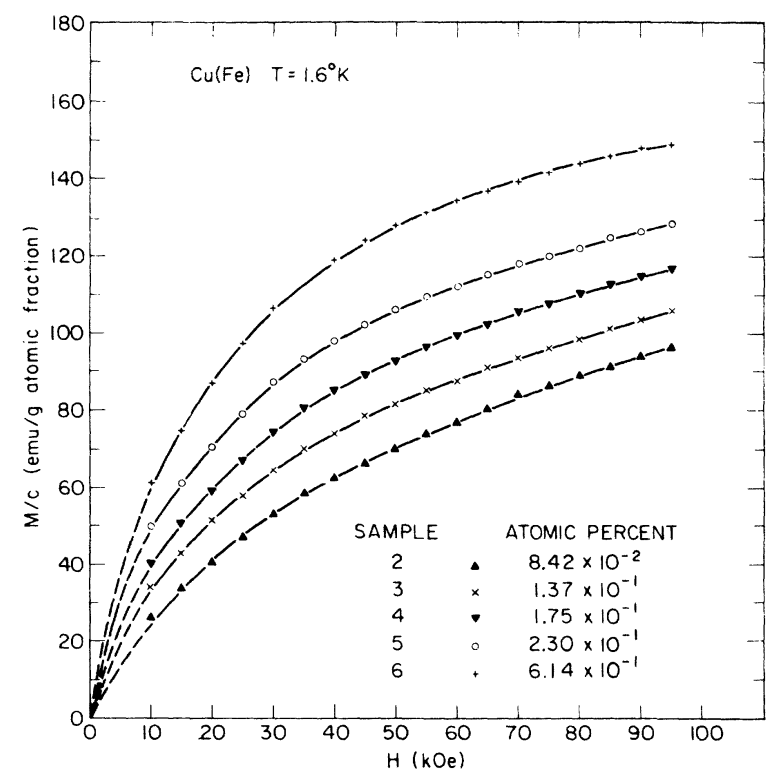

FIG. 5. High-field $M / c$ vs field for $\mathrm{Cu}(\mathrm{Fe})$ alloys at $T=1.6^{\circ} \mathrm{K}$ (unannealed samples).

purities in a nonmagnetic host. In this approximation an internal field is assumed to arise from an indirect exchange interaction via the conduction electrons, between magnetic impurities. Most attempts to treat the problem of interacting moments in metals assume the interaction given by Ruderman and Kittel, ${ }^{24}$ Kasuya, ${ }^{25}$ and Yosida ${ }^{26}$ (RKKY) has the approximate form

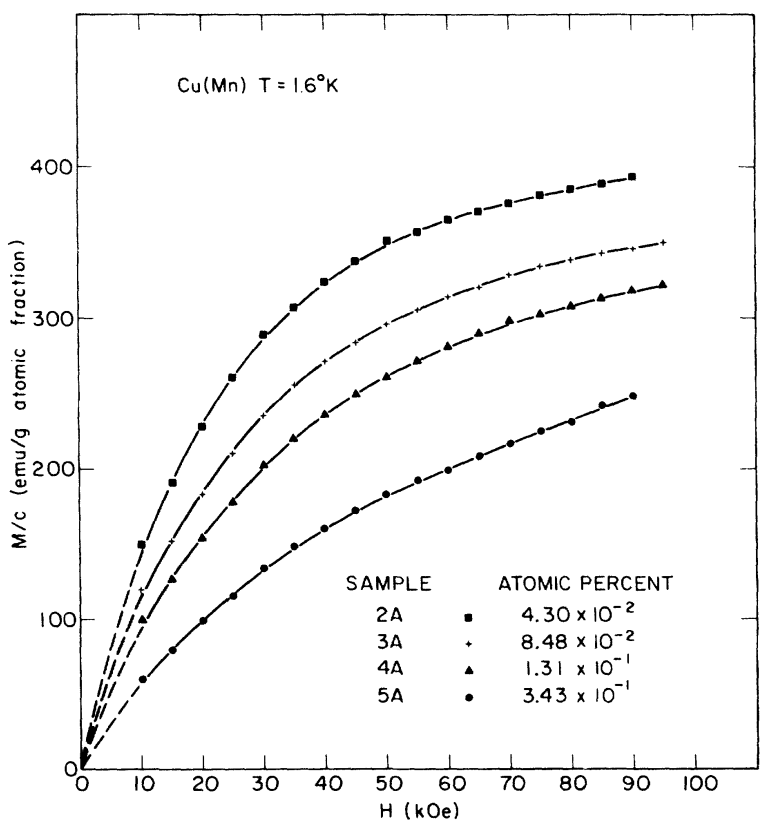

FIG. 6. High-field $M / c$ vs $H$ for $\mathrm{Cu}(\mathrm{Mn})$ alloys at $T$ $=1.6^{\circ} \mathrm{K}$. 


$$
E_{i j}=\alpha \vec{\mu}_{i} \cdot \vec{\mu}_{j} \frac{\cos \left(2 k_{F} r_{i j}\right)}{r_{i j}^{3}}
$$

Here $\alpha$ is a constant, $\gamma_{i j}$ is the distance between moments $\vec{\mu}_{i}$ and $\vec{\mu}_{j}$, and $k_{F}$ is the Fermi wave vector. The most recent works of $\mathrm{Klein}^{15}$ have relevance to the present work and will be described here briefly for future reference. It is assumed by Klein that the alloy may be considered as a random distribution of Ising-model spins in a nonmagnetic host. Furthermore, the calculation considers only the limiting case as $c \rightarrow 0$ (introducing an error of order $c^{2}$ ), and that long-range magnetic order is absent in the solid. For such a system, the interaction Hamiltonian between magnetic moments $\mu_{i}$ has the form

$$
H=\sum_{i<j} v_{i j} \mu_{i} \mu_{j},
$$

where the moments have values of \pm 1 and the RKKY interaction potential is of the form

$$
v_{i j}=a\left(\cos k_{F} r_{i j} /\left|\cos k_{F} r_{i j}\right|\right) r_{i j}^{-3} .
$$

Here $a$ is the interaction strength. The RKKY interaction is incorporated into the theory using the assumption that $k_{F} \bar{R} \gg 1$, where $\bar{R}$ is the average distance between magnetic impurities. Under the se conditions, Eq. (4.3) varies sufficiently rapidly that an impurity $j$ is, on the average, just as likely to experience a positive as a negative potential from impurity $i$. Equation (4.3) is then approximated by

$$
v_{i j}=+a / r_{i j}^{3} \text { or }-a / r_{i j}^{3},
$$

each with probability $\frac{1}{2}$.

Using a mean-random-field (MRF) approximation, a temperature- and concentration-dependent distribution $P(\bar{H})$ (probability distribution of the random molecular field $\bar{H}$ ) is determined. The thermodynamic variables of the system are obtained by integrating the expression for the thermodynamic variable of a single spin in a molecular field $\bar{H}$ over the distribution for all fields. $P(\bar{H})$ is characterized by a temperature- and concentrationdependent width $\Delta$. In the $T=0$ limit, $\Delta \rightarrow \Delta(\infty)$ $=\gamma c$, where $\gamma=\frac{8}{3} \pi^{2}|a| n_{0}, a$ is the interaction strength between impurities at a distance of one lattice constant, and $n_{0}$ is the number of sites per unit cell ( $n_{0}=4$ for an fcc lattice).

The results, when applied to the magnetic properties at very low temperatures, give a magnetic susceptibility which is independent of impurity concentration and a temperature-dependent susceptibility characterized by a maximum where the temperature of the maximum, $T_{\max }$, is proportional to impurity concentration. Similarly, the very-low-temperature specific heat is independent of impurity concentration and approximately linear with $T$.

Recently, Klein ${ }^{15}$ has extended the above theory to include the presence of an external magnetic field $H_{\text {ext }}$. In this case $\Delta$ also depends on external magnetic field. In particular, the theory gives a low-temperature expression for the magnetization as a function of $T, H_{\text {ext }}$, and $c$. Low-temperature results relevant to this work, taken from Ref. 15, are given below:

$$
\begin{aligned}
& \Delta=\gamma c\left[1-\frac{\ln 4}{\pi} \frac{k_{B} T}{\gamma c}-\left(\frac{\ln 4}{\pi} \frac{k_{B} T}{\gamma c}\right)^{2}\right], \\
& \frac{\chi_{i}(T)}{N_{0} \mu_{B}^{2} / \pi}=\frac{2}{\gamma}\left[1+\frac{\ln 4}{\pi} \frac{k_{B} T}{\gamma c}\right. \\
& \left.-\left(\frac{\pi^{2}}{12}-\frac{2 \ln ^{2} 4}{\pi^{2}}\right)\left(\frac{k_{B} T}{\gamma c}\right)^{2}\right] \quad\left(\gamma c \gg k_{B} T\right) .
\end{aligned}
$$

$\chi_{i}(T)$ is the susceptibility per mole of alloy and $N_{0}$ is Avogadro's number. A useful relation for estimating $\gamma$ is

$$
k_{B} T_{\max } \simeq 0.4 \Delta(\infty)=0.4 \gamma c .
$$

From Ref. 15, the low-temperature field-dependent magnetization is given by

$$
\begin{aligned}
& M= \frac{2}{\pi} N c \mu_{B}\left\{\tan ^{-1} y_{0}+\frac{2 y_{0} \ln 2}{\pi\left(1+y_{0}^{2}\right)^{2}}\left(\frac{k T}{\Delta(\infty)}\right)\right. \\
&+\left(\frac{k T}{\Delta(\infty)}\right)^{2}\left(\frac{y_{0}}{1+y_{0}^{2}}\right)\left[\left(\frac{2 \ln 2}{\pi\left(1+y_{0}^{2}\right)}\right)^{2}\right. \\
&\left.\left.\times \frac{4+3 y_{0}^{2}}{1+y_{0}^{2}}-\frac{\pi^{2}}{12}\right]+O\left(T^{3}\right)\right\},
\end{aligned}
$$

where $N$ is the number sites in the crystal and $y_{0}$ $=\mu_{B} H_{\text {ext }} / \Delta(\infty)$.

\section{B. Experimental Evidence for a Molecular-Field Model}

Low-temperature measurements at $T \sim 0.1{ }^{\circ} \mathrm{K}^{9}$ indicate that for $\mathrm{Cu}(\mathrm{Mn})(0.0186<c<0.196$ at. \%) the susceptibility tends to a concentration-independent limit of $\chi_{l}=1.0 \times 10^{-3} \mathrm{emu}$ per mole of alloy. Specific-heat data ${ }^{27}$ at liquid-helium temperatures for $\mathrm{Cu}(\mathrm{Mn})(0.5<c<10.0$ at. \%) indicate the specific heat due to impurities to be nearly linear in $T$ and nearly independent of $\mathrm{Mn}$ concentration. Dreyfus et al. ${ }^{12}$ compared the low-temperature specific heat and susceptibility for $\mathrm{Cu}(\mathrm{Mn})$, $\mathrm{Au}(\mathrm{Fe})$, and $\mathrm{Cu}(\mathrm{Fe})$ with theoretical relations between $\chi_{i}$ and heat capacity $\Delta C$ at $T \simeq 0$. Excellent agreement was found for $\mathrm{Cu}(\mathrm{Mn})$ and $\mathrm{Au}(\mathrm{Fe})$.

In addition Klein and Shen ${ }^{28}$ found good agreement with the MRF theory for $\chi_{i}(T)$ data for $\mathrm{Au}$ alloys $[\mathrm{Au}(\mathrm{Fe}), \mathrm{Au}(\mathrm{Cr})$, and $\mathrm{Au}(\mathrm{Mn})]$ in the temperature range $T_{\max }<T<2 T_{\max }$. In contrast, $\mathrm{Cu}(\mathrm{Fe})$ susceptibility measurements at $T \sim 0.5^{\circ} \mathrm{K}^{12}$ 
show a strong concentration dependence $(0.001$ $<c<1.0$ at. \%) with an abrupt change in this dependence at approximately 0.1 at. $\%$. Specific-heat data for $\mathrm{Cu}(\mathrm{Fe})^{29}$ also indicate a concentration dependence to $T \simeq 0.4^{\circ} \mathrm{K}$. This evidence lends support to the qualitative description of the randommolecular-field theory, at least for the $\mathrm{Cu}(\mathrm{Mn})$ and several Au-alloy systems.

\section{Local-Environment Model and Impurity Magnetization}

In a local-environment model of impurity magnetization, it is assumed that the magnetic properties of an impurity are, to a large extent, determined by the number and type of atoms in the immediate vicinity of the impurity. This approach was used by Jaccarino and Walker ${ }^{30}$ to explain how Co atoms (nonmagnetic in $\mathrm{Rh}$ ) form a moment in $\mathrm{Rh}(\mathrm{Pd})$ as the $\mathrm{Pd}$ concentration is increased. It was found that Co forms a moment only when it has two or more Pd atoms in its nearest-neighbor sites. The local conditions for moment formation have been studied in other systems, including $\mathrm{Fe}$ or $\mathrm{Co}$ in $\mathrm{Al},{ }^{31-33} \mathrm{~V}$ in $\mathrm{Au},{ }^{34-37}$ $\mathrm{Co}$ in $\mathrm{Au}^{38}$ or $\mathrm{Cu},{ }^{39}$ and $\mathrm{Ni}$ in $\mathrm{Cu} .{ }^{40,41}$

Apart from the problem of forming impurity moments, the magnetization of a given moment may also depend on its local environment, as indicated by the recent study of $\mathrm{Cu}(\mathrm{Fe})$ by Tholence and Tournier. ${ }^{13}$ The relationship between their work and the present work will be discussed in detail in Sec. V.

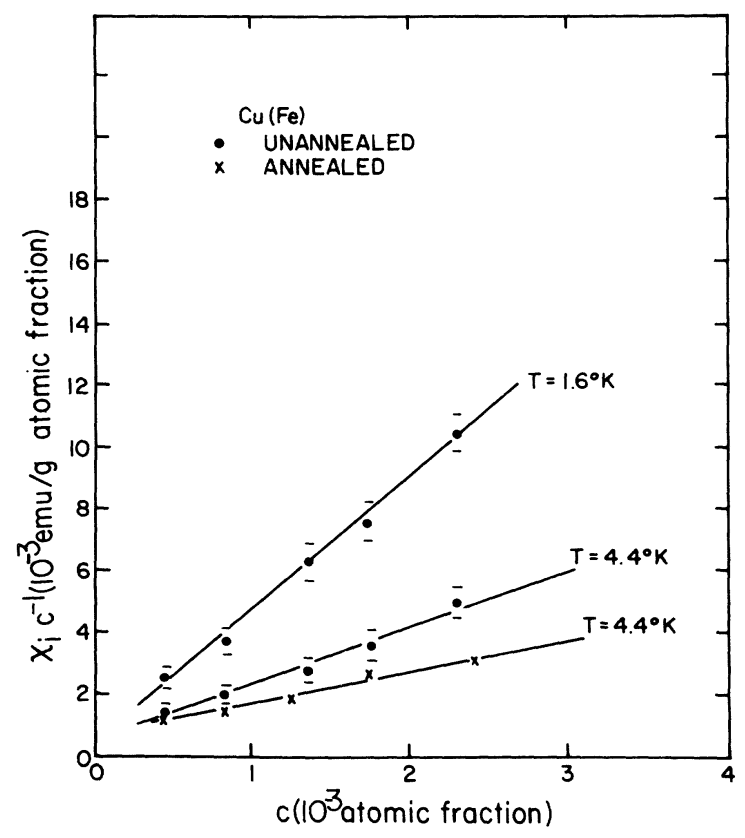

FIG. 7. $\chi_{i} / c$ vs $c$ for lower concentration $\mathrm{Cu}(\mathrm{Fe})$ alloys at 1.6 and $4.4^{\circ} \mathrm{K}$.
The connection between bulk measurements of magnetization and local-environment model is made through the concentration dependence of the bulk magnetization. It is assumed that the $N_{i}$ impurity atoms go into the $N$ lattice sites randomly and that next-nearest-neighbor interactions are unimportant. Then the probability that a given impurity atom has $\eta$ nearest-neighbor impurity atoms is given by

$$
P_{\eta}(c)=Z ! c^{\eta}(1-c)^{Z-\eta} /[(Z-\eta) ! \eta !],
$$

where $Z$ is the number of nearest-neighbor sites for a given lattice ( $Z=12$ for fcc) and $c=N_{i} / N$ is the impurity concentration. The number of "clusters" containing one, two, and three impurity atoms is given by $N_{1}=N c P_{0}, N_{2}=N c P_{1} / 2$, and $N_{3}$ $=N c P_{2} / 3$. If we associate a magnetization with each type of local environment, then the total impurity magnetization is given approximately by

$$
M=N_{1} M_{1}+N_{2} M_{2}+N_{3} M_{3}+\ldots \text {. }
$$

Here $M_{1}, M_{2}$, and $M_{3}$ are the magnetizations associated with a spin cluster containing one, two, and three impurity atoms, respectively.

\section{V. $\mathrm{Cu}(\mathrm{Fe})$ DISCUSSION}

A. General Features of the Magnetization

As discussed in Sec. IV, the experimental evidence indicates that $\mathrm{Cu}(\mathrm{Fe})$ has concentration dependences differing from tho se of $\mathrm{Cu}(\mathrm{Mn})$ and $\mathrm{Au}(\mathrm{Fe})$. For example, Fig. 2 shows that for $\mathrm{Cu}(\mathrm{Mn}) \chi_{i} \propto c$ above $\sim 10^{\circ} \mathrm{K}$, while for $\mathrm{Cu}(\mathrm{Fe})$ this is not the case to $40{ }^{\circ} \mathrm{K}$ (or even to $300^{\circ} \mathrm{K}^{42}$ ), as seen in Fig. 3. The magnetic interactions in $\mathrm{Cu}(\mathrm{Fe})$ are therefore considerably stronger than those in $\mathrm{Cu}(\mathrm{Mn})$ in the sense that they cannot be broken up even by rather high-temperature thermal fluctuations. Furthermore these interactions are ferromagnetic in nature, since $c / \chi_{i}$ decreases as $c$ increases. Figure 7 shows these interaction effects for the lower-concentration samples at 1.6 and $4.4^{\circ} \mathrm{K}$. At first glance this figure suggests that $\chi_{i} / c=a+b c$, which would suggest that the susceptibility contains two terms; one due to isolated iron atoms (ac) and one due to some type of iron clusters $\left(\mathrm{bc}^{2}\right)$. The figure then implies that the re is very little temperature dependence between 1.6 and $4.2{ }^{\circ} \mathrm{K}$ for the isolated iron susceptibility ( $a \simeq$ const) and that the cluster term (the slope of the lines) is considerably larger for the unannealed than for the annealed samples. It should be pointed out that the scatter in the $\chi_{i}$ data as well as the relatively narrow range of concentrations shown in Fig. 7 argue against saying that there is definitely simply a linear and quadratic concentration term in $\chi_{i}$. However, we shall return in Sec. V C to a discussion of the concentration 


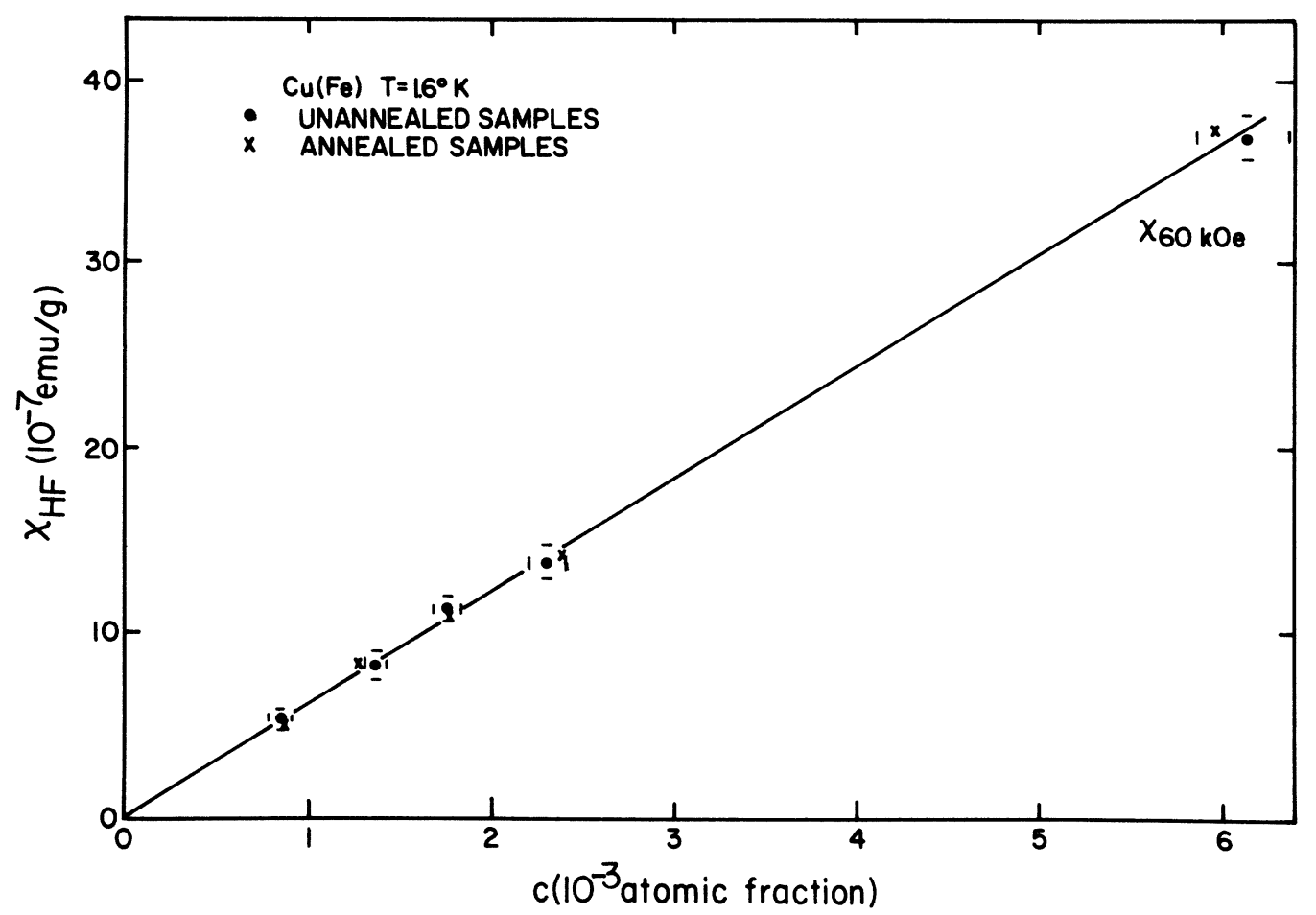

FIG. 8. High-field susceptibility vs $c$ for the two sets of $\mathrm{Cu}(\mathrm{Fe})$ samples at $1.6^{\circ} \mathrm{K}$.

dependence in connection with our more accurate high-field-magnetization data.

In contrast to the differences seen in the annealed and unannealed samples (Fig. 7), an interesting similarity between the two sets of samples is seen in Fig. 8. Namely, the high-field susceptibility $\chi_{\mathrm{HF}} \equiv(d M / d H)_{\mathrm{HF}}$ at $60 \mathrm{kOe}$ is the same for both sets of samples. This indicates that $\chi_{\mathrm{H} F}$ is dominated by the linear term in $c$ and that annealing has little effect on the high-field behavior. Also of interest in Fig. 8 is the apparently negligible effect of the magnetic "ordering" (Fig. 4) on the value of $\chi_{\mathrm{HF}}$ for the highest-concentration sample. That $\chi_{\mathrm{HF}}$ for this sample below its ordering temperature lies on the same line as the lowerconcentration samples, suggests either that the ordering involves only a small fraction of the spins, or else that at very high fields the interactions responsible for the ordering are overpowered by the interactions between the spins and the external field.

A final general feature of the results that should be mentioned is the low-temperature flattening of $\chi$ for the 6140-ppm sample. Thus, in Fig. 3, $c / \chi_{i}$ increases above the Curie-Weiss extrapolation below $\sim 10^{\circ} \mathrm{K}$, behavior reminiscent of the higherconcentration $\mathrm{Cu}(\mathrm{Mn})$ samples as seen in $\mathrm{Fig} .2$. This indicates that there are some manifestations of the antiferromagnetic interactions which lead to typical spin-glass behavior, but, in addition, there are ferromagnetic interactions present which complicate the normal situation.

In attempting to build a model to explain the above results it will be useful to consider in Secs. V B-D the magnetization in three different concentration regions: the extreme-dilution limit, the magnetic-interaction region, and the "spin-glass" region. It will be seen that the boundaries between these regions are not sharp, but it will facilitate the discussion to begin with the simplest situation (extreme dilution) and then proceed to the complex ("spin-glass") region.

\section{B. Extreme Dilution Region}

This is defined to be the $c \lesssim 300-p p m$ region, which corresponds to the region in which Tholence and Tournier (TT) were able to construct a model for these alloys. ${ }^{13}$ During the course of our experiments on higher-concentration alloys, TT showed that for $c \lesssim 300 \mathrm{ppm}$ the magnetization $M(H)$ to $\sim 65$ kOe contained terms linear and quadratic in $c$. They found that the susceptibility could be expressed as

$$
\chi=C_{1} /\left(T+T_{K_{1}}\right)+C_{2} /\left(T+T_{K_{2}}\right)
$$

where $C_{1} \propto c, C_{2} \propto c^{2}, T_{K_{1}} \simeq 29^{\circ} \mathrm{K}$, and $0 \lesssim T_{K_{2}}$ $\lesssim 5^{\circ} \mathrm{K}$. Since the $c^{2}$ magnetization term saturated at $60 \mathrm{kOe}$, it was possible to measure both the saturation moment and density of the magnetic en- 
tities associated with the $c^{2}$ term in $M(H)$. It was found that the spin associated with these entities was $S_{2} \simeq 2.7$, which is approximately $2 S_{1}$, where $S_{1} \simeq \frac{3}{2}$ is the spin of an isolated iron impurity in copper. This, plus the $c^{2}$ dependence, led to the reasonable association of the $c^{2}$ term in $M(H)$ with pairs of $\mathrm{Fe}$ atoms which are ferromagnetically coupled. The density of pairs was found to be $130 c^{2}$. Since this pair density is so much higher than that expected for random occupation of impurities on nearest-neighbor sites $\left(6 c^{2}\right)$, TT assumed that the pairs were coupled at long range via the RKKY interaction. In that case the total number of pairs (including the antiferromagnetically coupled atoms) would be $260 c^{2}$ and the number of Fe atoms in pairs would be $520 c^{2}$. This density then leads to an average volume per $\mathrm{Fe}$ atom in a pair from which TT suggested a critical radius $(\simeq 11 \AA)$ for pair formation. This idea of pair formation by RKKY coupling has been adopted by several other workers who have observed interaction effects in $\mathrm{Cu}(\mathrm{Fe})$ alloys at low temperatures. ${ }^{10,14}$

From the viewpoint of the Kondo effect, TT made an important contribution because they showed for the first time that interaction effects, which had confused much of the earlier experimental and theoretical work, were not negligible in $\mathrm{Cu}(\mathrm{Fe})$-even down to the 10-ppm range. From the viewpoint of the present work, however, it is the magnetic interactions which are of major interest and we shall focus attention in the following on the nature of the interaction leading to pair formation and possibly to spin-glass transitions.

\section{Magnetic Interaction Region}

In the concentration region from $\sim 500$ to $\sim 3000$ ppm the magnetization cannot be analyzed simply in terms of $c$ and $c^{2}$ contributions. This is seen already for the 600-ppm sample of TT, which deviates from the straight line $M / c$-vs- $c$ plot for the lower- $c$ samples. The reasons for this can be understood by referring to the local-environment model of Sec. IV C. Suppose that a given Fe atom has $z$ sites in its neighborhood which, if occupied by another $\mathrm{Fe}$ atom, form a magnetic entity called a pair. Suppose also that the concentration is not so high that clusters of three or more have to be considered. Then, from Eqs. (4.9) and (4.10),

$$
M=c N(1-c)^{2} M_{1}+\frac{1}{2} z c^{2} N(1-c)^{z-1} M_{2},
$$

where the coefficients of $M_{1}$ and $M_{2}$ are the number of isolated $\mathrm{Fe}$ atoms and the number of pairs of Fe atoms, respectively. Then

$$
M=\left[N c(1-c)^{2}\right]\left(M_{1}+\frac{c z}{2(1-c)} M_{2}\right) \text {. }
$$

Defining a new quantity $\mu$ and noting that $c \ll 1$, we

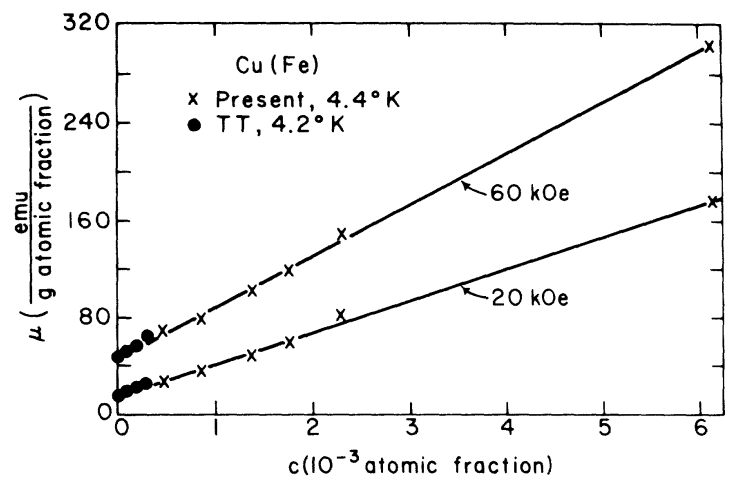

FIG. 9. Concentration dependence of $\mu$ (see text) at 20 and $60 \mathrm{kOe}$. Crosses are for unannealed alloys. The very low concentration data of TT are also shown.

have finally

$$
\mu \equiv M / N c(1-c)^{x} \simeq M_{1}+\frac{1}{2} z c M_{2} \text {. }
$$

Since $z$ is of order $10^{2}$, the $(1-c)^{2}$ factor becomes appreciable when $c \gtrsim 500 \mathrm{ppm}$ and it is this circumstance which destroys the straight-line plot of $M / c$ vs $c$ 。 In the $c \rightarrow 0$ limit this analysis clearly approaches that of TT. We have analyzed our lowtemperature and high-field data with Eq. $(5,1)$ in the following way. $\mu(H, c)$ was fitted to Eq. (5.1) with a computer program which determined the parameter $z$ at each $H$ and $T$. Fits were performed with our data alone and with those of TT to $60 \mathrm{kOe}$. This was done to test the two sets of data for consistency and for sensitivity to sample-preparation procedures. Figure 9 shows a typical example of such a fit. It is seen that our high- $c$ data match approximately with the low-c data of TT, but that our slope, corresponding to the pair term, is somewhat smaller; this is true for both the $\mathrm{Cu}(\mathrm{Fe})$ and $\mathrm{Cu}(\mathrm{Fe}) \mathrm{A}$ alloys. An example of the field dependence to $90 \mathrm{kOe}$ of $M_{1}$ and $M_{2}$ (derived from our data alone) is shown in Fig. 10, along with curves taken from TT. Again, there is semiquantitative agreement in both $M_{1}$ and $M_{2}$ and, in particular, the saturation value of $M_{2}(\sim 500 \mathrm{emu} / \mathrm{g})$ leads to a spin value $S_{2} \simeq 2.9$ per pair, which is similar to the value determined by TT. Fits with the $\mathrm{Cu}(\mathrm{Fe}$ ) (unannealed) alloys gave similar $S_{2}$ values, but both sets of samples showed smaller- $z$ values than that suggested by TT $(z=260)$. For example, at $1.6{ }^{\circ} \mathrm{K}$ the fits at $30 \mathrm{kOe}$ for the $\mathrm{Cu}(\mathrm{Fe})$ and $\mathrm{Cu}(\mathrm{Fe}) \mathrm{A}$ samples gave $z=170$ and 130, respectively. The accuracy of these $z$ values is not better than $15-20 \%$, but it is certainly true that the number of pairs in the $\mathrm{Cu}(\mathrm{Fe})$ samples is larger by a factor of $\sim \frac{3}{2}$ than in the $\mathrm{Cu}(\mathrm{Fe}) \mathrm{A}$ samples-as is seen explicitly also in Fig. 7.

To get further information on the nature of the magnetic pairs, we now consider recent Mössbauer 


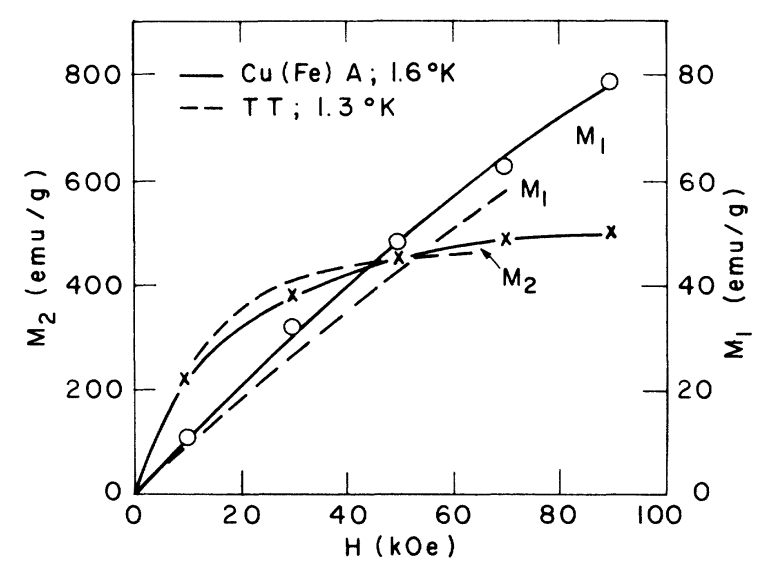

FIG. 10. Field dependence of the magnetization of isolated $\left(M_{1}\right)$ and pairs $\left(M_{2}\right)$ of Fe atoms. Data of TT to $\sim 60$ kOe ar e shown for comparison.

experiments on $\mathrm{Cu}(\mathrm{Fe})$ alloys in the range $c$ $\leqslant 5000 \mathrm{ppm}$. Although early work was done by Bennett and Swartzendruber, ${ }^{43}$ Window, ${ }^{44}$ and others, we shall consider here mainly the very recent work of Campbell et al. ${ }^{45}$ and Knauer, ${ }^{46}$ which covers more comprehensively the concentration region of interest. The main features of the results are as follows. At high temperatures (e.g. , $300^{\circ} \mathrm{K}$ ) the Mössbauer spectra for dilute alloys consist of a single line with two smaller lines which are just resolvable on either side of the "central" line. As the concentration increases, the smaller lines increase in area relative to the central line. The interpretation given to these spectra is that the central line is due to isolated $\mathrm{Fe}$ atoms and the additional lines are due to $\mathrm{Fe}$ atoms in Fe clusters. Campbell et al. ${ }^{45}$ analyzed their data by fitting them to a single Lorentzian line of relative area $A_{0}$ (the central line) and a number of doublets of relative area $A_{1}, A_{2}$, etc. In addition to the areas, the fit gave the isomer shifts and quadrupole splittings for the various doublets. The results showed that for the various doublets, the isomer shift and quadrupole splitting were approximately constant from sample to sample. This, along with the concentration dependence of the areas, suggested that the single line was due to isolated $\mathrm{Fe}$ atoms and succeeding doublets of decreasing intensity were due to the variation in electric field gradient at an iron site produced by the charge asymmetry of clusters of 2,3 , and 4 iron atoms. The isomer shift increased monotonically from $\sim 0.0 \mathrm{~mm} / \mathrm{sec}$ for the first doublet to $\sim-0.14 \mathrm{~mm} / \mathrm{sec}$ for the third doublet, and this is further reason for identifying each doublet with an increasing number of nearest-neighbor iron atoms; the isomer shift in pure iron is -0.22 $\mathrm{mm} / \mathrm{sec}$.

Additional information on local-environment effects in these alloys was determined by the lowtemperature work of Window. ${ }^{44}$ He fitted the $1.4^{\circ} \mathrm{K}$ data for several samples in the magnetically "ordered" state with a hyperfine-field distribution function $P(H)$, which showed peaks at $\sim 80,150$, $240 \mathrm{kOe}$, etc. The concentration dependence of the $P(H)$ curves indicated that there was a hyperfine field of $80 \mathrm{kOe}$ for an isolated iron atom, $150 \mathrm{kOe}$ for an iron atom with one iron neighbor, etc., and this interpretation was quite consistent with the madel discussed above.

It is now possible to make an informative connection between the above Mössbauer results and the present magnetization results. As determined by the Mössbauer effect, the ratio of the number of iron atoms in pairs to the number of isolated ones is $A_{1} / A_{0}$, in the notation used above. Thus the Mössbauer ratio of iron pairs to isolated iron atoms is

$$
R^{M} \equiv A_{1} / 2 A_{0}
$$

These areas are tabulated by Campbell et al. ${ }^{45}$ and $R^{N}$ is plotted in Fig. 11. Though Knauer did not analyze his data in the same manner as Campbell $e t a l .$, it is possible to derive values of $R^{M}$ from his data ${ }^{46}$ and these are also shown in Fig. 11. Knauer also showed that data obtained from single-crystal and polycrystalline samples were identical so that iron clustering at grain boundaries is insignificant for alloys with $c \lesssim 5000 \mathrm{ppm}$. The magnetization analysis of Eq. (5.1) gives

$$
R=\frac{1}{2} z c
$$

for the ratio of pairs to singles, and Fig. 11 shows curves for $z=260,170$, and 130 relevant to various sets of samples as labeled on the figure. Since the slope of the curves is proportional to the pair concentration, Fig. 11 has the following implications: (i) All of the magnetization and Mössbauer samples have many more pairs than would be expected on the basis of a perfectly random nearestneighbor occupation of iron atoms on the fcc lattice (for which $R=6 c$ ). (ii) All of the samples quenched either from the melt or from the high-temperature solid-solution phase have a higher pair density than the cold-rolled samples of Campbell et al. That is, the cold-rolled samples are much closer to random alloys than the quenched samples-a conclusion previously reached by Window and shown by the data in Table I of Campbell et al. The randomizing effect of cold rolling is greater for the higher-concentration alloys than for the lower-concentration ones in that the fractional deviation from the $R=6 c$ (random) curve is smaller at the higher concentrations. (iii) Among the quenched magnetization and Mössbauer samples there is a continual increase in the pair density as one goes from our solution-annealed samples to the solution-an- 


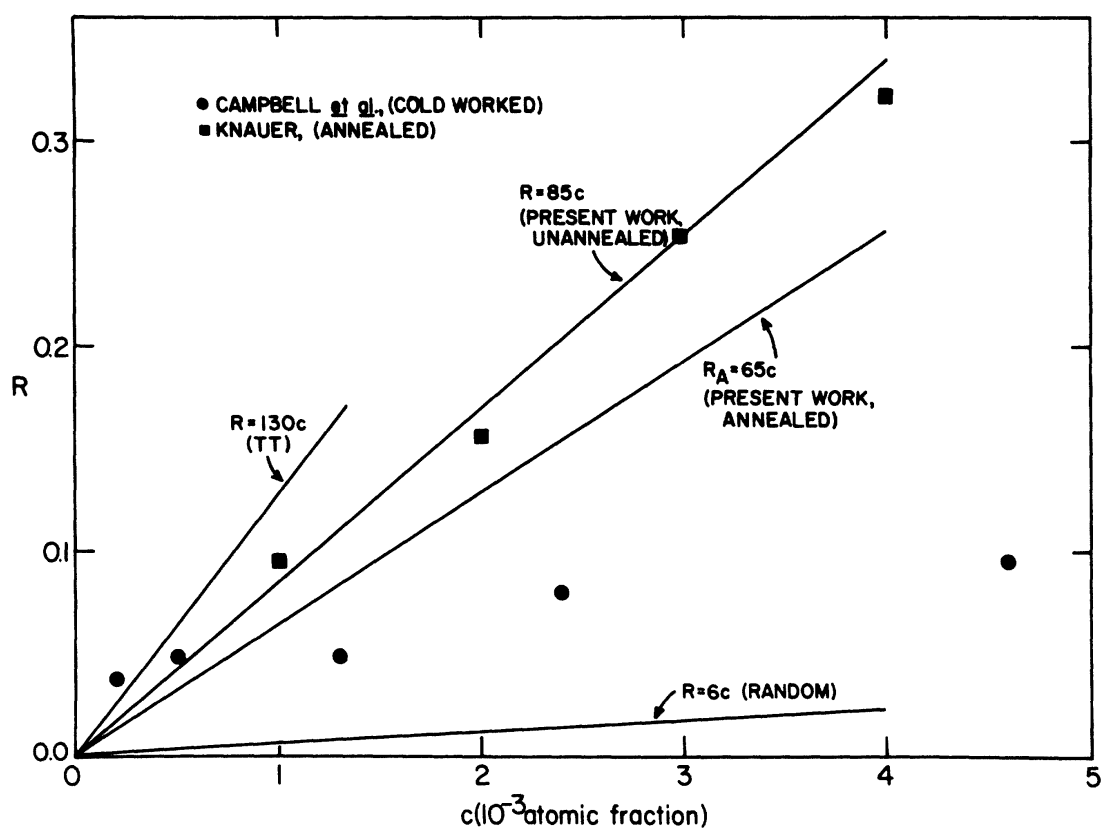

FIG. 11. Concentration dependence of the ratio of pair to isolated $\mathrm{Fe}$ atom concentration. The data are taken from the present work and various other sources as explained in text.

nealed Mössbauer samples and from our chill-cast samples to the chill-cast samples of TT.

Points (ii) and (iii) above indicate that the pair density is not an intrinsic property of atomic or electronic properties of the $\mathrm{Cu}(\mathrm{Fe})$ system, but rather is determined by structural effects which are strongly influenced by sample-preparation procedures. This ought not be too surprising when it is recalled that these alloys are supersaturated. The results generally are inconsistent with the supposition ${ }^{13}$ that the pairs result from long-range RKKY interactions between randomly placed spins. A critical coupling distance such as $11 \AA$, which is calculated directly from the pair density, clearly must be a function only of the parameters that enter the RKKY interaction-one of which is certainly not the structural state of the sample. A more reasonable explanation of the variation in pair density observed in the various sets of samples is that there is a strong tendency for atomic short-range ordering or clustering between the iron atoms, and that the degree of clustering is strongly influenced by quench rate, cold work, and other variables of the sample-preparation methods. Since the "collision probability" for two impurity atoms, which at high temperatures are mobile in the sample, would be expected to be proportional to $c^{2}$, one would expect a $c^{2}$ term in the magnetization due to the magnetic properties of what might be called "diatomic Fe molecules" dissolved in the $\mathrm{Cu}$ matrix. Such nearest-neighbor magnetic pairs clearly should be treated from a self-consistent spin-polarized cluster model rather than from the RKKY viewpoint. In fact, Mössbauer and magnetization studies of the type we have been discussing appear to have great promise for studying such impurity clusters dissolved in metals.

\section{Spin-glass Transitions}

One of the original motivations for the present work was to understand why $\mathrm{Cu}(\mathrm{Fe})$ apparently did not exhibit magnetic interactions and spin-glass transitions (susceptibility and resistivity maxima) similar to those seen in $\mathrm{Cu}(\mathrm{Mn}), \mathrm{Au}(\mathrm{Fe})$, and other alloys. There had been speculation by Klein, ${ }^{15}$ for example, that perhaps the spin correlations associated with the Kondo effect interfered with the RKKY coupling below the Kondo temperature $\left(\sim 10^{\circ} \mathrm{K}\right)$. Typically, the spin-glass transitions occur at a temperature $T_{\max } \simeq\left(20^{\circ} \mathrm{K} /\right.$ at. \% $)$ c. Thus for a 0.5 -at. $\%$ alloy one would expect $T_{\max } \simeq 10^{\circ} \mathrm{K}$. We have observed a type of magnetic "ordering" in our 0.6 -at. $\%$ alloy, remanence below $3.5^{\circ} \mathrm{K}$, as shown in Fig. 4. Our concentration and temperature ranges, however, made it impossible to determine the concentration dependence of the ordering temperature. An interesting clue concerning the nature of the ordering as manifested in the remanence is that the ratio of the annealed and unannealed remanence values is $M_{r}^{A} / M_{r}=81 / 105$ $=0.77$. This number is very close to the ratio $P$ of the pair densities for the annealed and unannealed samples; from Eq. (5. 3) $P=z^{A} / z \simeq 130 / 170$ $=0.76$, where $z^{A}$ and $z$ refer to annealed and unannealed values of the coupling parameter of Eq. (5.1). This correspondence rather strongly suggests that the remanence is due to the pairs which can be aligned by a field of order $10 \mathrm{kOe}$, but 
which experience an interaction which keeps them aligned when the external field is reduced to zero. It is difficult to understand precisely the mechanism for this remanence but it may be due to longrange (RKKY-type) interactions between the pairs or perhaps interactions between the pairs and the "isolated" spins which, themselves, are unable to flip thermally at low temperatures. ${ }^{47}$ Additional evidence on this point is contained in the work of Hirschkoff $e t$ al.,${ }^{10}$ who found at very low temperatures $\left(\sim 0.01-0.4^{\circ} \mathrm{K}\right)$ that there was an apparent ordering of the magnetic pairs at a temperature $T_{0}$ proportional to $c^{2}$. At such low temperatures the magnetization contribution from the isolated spins is small. Since the typical spin-glass behavior in which $T_{\max } \propto c$ is explicable in terms of the RKKY coupling of single spins, it is reasonable to associate the observed ordering temperature $T_{o}$ with RKKY-type ordering between pairs whose concentration is proportional to $c^{2}$.

Finally, it is possible to understand the spinglass transition data of Svensson ${ }^{48}$ in terms of the discussion of Sec. VA-C. Svensson found re-sistivity and susceptibility maxima in $\mathrm{Cu}(\mathrm{Fe})$ alloys but only if the samples were strongly cold worked. The temperatures of these transitions are shown in Fig. 12 along with other indications of transitions from magnetization and heat-capacity data. This behavior is quite reasonably explained in terms of our previous discussion. It was seen that cold work breaks up iron pairs and other larger clusters, leading to a much more random array of impurity spins. Such an array is probably similar to that which exists in the typical spin glasses $\mathrm{Cu}(\mathrm{Mn})$ and $\mathrm{Au}(\mathrm{Fe})$, which are good solidsolution alloys over a relatively wide concentration range. The reason for the absence of the suscepti-

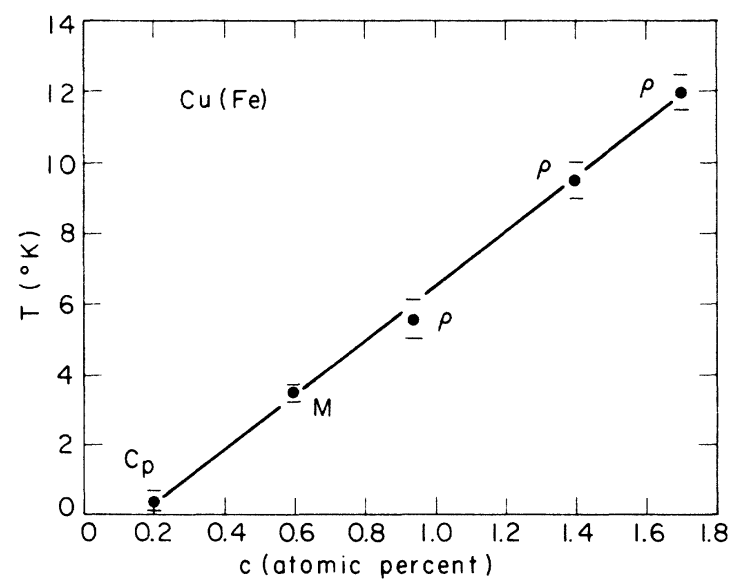

FIG. 12. Concentration dependence of the apparent "ordering" temperature as deduced from anomalies in heat capacity (Ref. 29), magnetization (present work), and resistivity (Ref. 48). bility maxima in Svensson's alloys which were not cold worked is likely the fact that in these samples there is a much larger relative number of pairs and larger clusters, in comparison with the isolated spins, than in the cold-worked alloys. Since the pairs and probably the larger clusters have very low Kondo temperatures (Weiss temperatures), the total susceptibility, which is dominated by these pairs or clusters, continues to rise as $T$ decreases-thus obliterating the more normal susceptibility maximum for which $T_{\max } \propto c$. At an extremely low temperature, the pairs order, leading to the transitions observed by Hirschkoff et al. ${ }^{10}$ for which $T_{0} \propto c^{2}$. This description is no doubt an oversimplification because, as seen in Fig. 12, there may be a complex transition region between the low-concentration pair-pair ordering seen in the non-cold-worked samples and the predominantly $\mathrm{Fe}-\mathrm{Fe}$ ordering seen in the cold-worked samples in which $T_{0}$ increases linearly with $c$. In this complex transition region it could well be the case that the ordering would have to be considered as due to a mixture of "isolated" and pair interimpurity interactions. An obvious experiment to test this would be to measure susceptibility of severely cold-worked samples to much lower concentrations and temperatures, to test the validity of the $T_{\max } \propto c$ relation for normal nonclustered spin glasses. One complicating feature of cold-worked alloys, however, is that the mean free path may be diminished somewhat, and this would be expected to decrease the coupling between spins. " On the other hand, in Svensson's alloys, in which the Fe impurity scattering was already high, the presence of the additional scattering due to the working seems not to have interfered significantly with the spin coupling; the spinglass transitions appear to be depending on concentration in the usual (mean-random-field) way, viz., $T_{\max } \propto c$.

E. Sub-Kondo Spin Polarization of a Magnetic Impurity at High Fields

Although it is somewhat peripheral to the main subject of this work, the data of Sec. V C contain information of interest for theoretical work on the Kondo problem. Namely, $M_{1}(H)$, the magnetization of an isolated Fe impurity, has been determined to $100 \mathrm{kOe}$ for temperatures much below $T_{K}$. There have been several theoretical calculations ${ }^{49,50}$ of the magnetization of the impurity spin and its associated "spin-compensation" cloud, but there appears to be some doubt that any of these are valid for any real system. ${ }^{51}$ For example, a calculation of single-impurity magnetization for $\mathrm{Cu}(\mathrm{Fe})$ was made by Bloomfield et al. ${ }^{50}$; but, unfortunately, they had to assume a spin value of $\frac{1}{2}$, which is known to be incorrect $\left(S \simeq \frac{3}{2}\right)$. It is there- 
fore impossible even to compare on the same plot the experimental results with their calculation because clearly the saturation value they would obtain is $\sim \frac{1}{3}$ of the correct value; also the field axis would have to be scaled to account for the fact that the "effective field" $\left(\mu_{B} H\right)$ is a function of the spin value assumed.

Figure 13 shows the experimental magnetization to high fields at $1.6^{\circ} \mathrm{K}$. The data agree to within $\sim 10 \%$ with those of $\mathrm{TT}$ in the region where they overlap $(H \leqslant 60 \mathrm{kG})$. Simply for the sake of comparison we have shown also in Fig. 13 two curves based on Brillouin functions for $S_{1}=\frac{3}{2}$ and $g=2$. The upper one corresponds to the magnetization of a free spin- $\frac{3}{2}$ particle and clearly does not fit the experimental data. Also a plot of $B_{3 / 2}$ is shown with an "effective" temperature of $T+29=30.6^{\circ} \mathrm{K}$. There is no a priori reason why this function should fit the data, but it at least has the virtue that as $H \rightarrow 0$, it leads to the experimentally observed susceptibility $\left(C_{1} / T+29\right){ }^{13}$ It is seen that this modified form of the Brillouin function does give a relatively good description of the data.

In conclusion, it would appear that further theoretical work on the magnetic polarization of a Kondo system in high fields is warranted.

\section{VI. $\mathrm{Cu}(\mathrm{Mn})$ DISCUSSION}

\section{A. General Features of $\mathrm{Cu}(\mathrm{Mn})$ Magnetization}

Figure 2 indicates that $\chi_{i}$ is approximately proportional to $c$ for temperatures above $10^{\circ} \mathrm{K}$ and

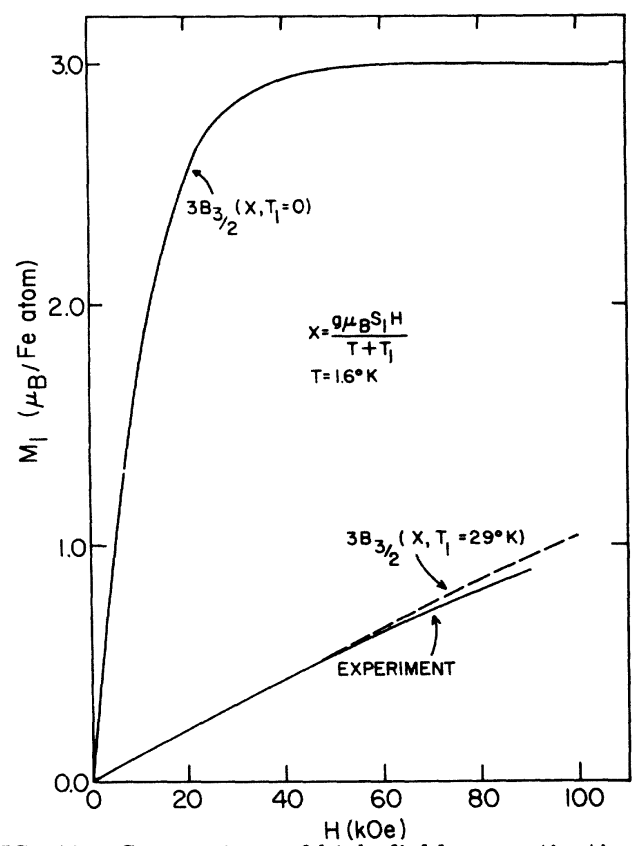

FIG. 13. Comparison of high-field magnetization of an isolated $\mathrm{Fe}$ spin at $1.6^{\circ} \mathrm{K}$ with free-spin and modified Brillouin functions, as explained in text. that significant deviations from linearity are observed for $T=1.6^{\circ} \mathrm{K}$. A plot of $\chi_{i} / c$ vs $c$ (Fig. 14) shows a decrease in the susceptibility per impurity as concentration is increased for $T$ less than $8^{\circ} \mathrm{K}$. A plot of $M / c$ vs $c$ for $T=1.6^{\circ} \mathrm{K}$ indicates similar behavior for fields up to $90 \mathrm{kOe}$. Figure 6 shows that for $T=1.6^{\circ} \mathrm{K}$ the impurity magnetization of the $\mathrm{Cu}(\mathrm{Mn})$ alloys studied is not saturated in a field of $95 \mathrm{kOe}$. The above results are all suggestive of antiferromagnetic interactions which have been observed ${ }^{6,7}$ in $\mathrm{Cu}(\mathrm{Mn})$ alloys containing greater than 1.0-at.\% $\mathrm{Mn}$ at liquid-helium temperatures.

Kouvel ${ }^{7}$ found that $\mathrm{Cu}(\mathrm{Mn})$ alloys containing 5-30-at. \% Mn exhibit a susceptibility maximum, and for $T<T_{\max }$ remanent magnetization is observed. This was interpreted as a magnetic state in which ferromagnetism and antifer romagnetism coexist. A qualitative description ${ }^{52}$ has been given in terms of clusters of antifer romagnetically coupled $\mathrm{Mn}$ atoms having a nonzero net moment. The formation of the clusters as $T$ approaches $T_{\max }$ accounts for the susceptibility maximum. At temperatures below $T_{\max }$, ferromagnetic ordering occurs between clusters giving the remanence observed. The susceptibility maximum is similarly described in a statistical model given by Klein and Brout. ${ }^{22}$ Kouvel $^{7}$ further suggests that nearestneighbor $\mathrm{Mn}$ atoms coupled antiferromagnetically are responsible for the antiferromagnetic behavior.

\section{B. Application of Mean-Random-Field Theory}

As indicated in Sec. IV there is experimental evidence that $\mathrm{Cu}(\mathrm{Mn})$ at very low temperatures obeys the MRF model. Since $\mathrm{Cu}(\mathrm{Mn})$ exhibits a susceptibility maximum and has a concentrationindependent low-temperature specific heat and susceptibility, it is possible to estimate the parameter $\Delta(\infty)=\gamma c$. Using this parameter, the initial susceptibility and field-dependent magnetization will be compared with theory.

Low-temperature susceptibility measurements by Dreyfus et al. ${ }^{12}$ give $T_{\max }=2.2^{\circ} \mathrm{K}$ for a 0.196-at.\% $\mathrm{Cu}(\mathrm{Mn})$ alloy. Using Eq. (4.7),

$$
k_{B} T_{\max } \simeq 0.4 \Delta(\infty)=0.4 \gamma c,
$$

one calculates $\gamma=2.8 \times 10^{3}{ }^{\circ} \mathrm{K} /$ at . fraction. The parameter $\gamma$ may also be estimated using the limiting susceptibility $\chi_{l}=1.0 \times 10^{3}$ emu per mole of alloy and the relation

$$
\gamma=2 N_{0} \mu_{B}^{2} S(S+1) / \chi(0) \pi .
$$

Here we have used Eq. (4.6), corrected for the Ising-model approximation by including the spin factor $S(S+1)$. For $\chi_{l} \simeq \chi(0)$ and $S=2$ we find $\gamma$ $=1.42 \times 10^{3} \mathrm{~K} /$ at . fraction. Since long-range order is not observed in $\mathrm{Cu}(\mathrm{Mn})$ for $T \geqslant T_{\max }$ the estimate of $\gamma$, using Eq. (4.7), is suggested by Klein ${ }^{53}$ and will be used here. Table III gives 
TABLE III. Expansion parameter $k_{B} T / \Delta(\infty)=k_{B} T / \gamma c$ for $\mathrm{Cu}(\mathrm{Mn})$ alloys.

\begin{tabular}{cccc}
\hline \hline & & \multicolumn{2}{c}{$k_{B} T / \Delta(\infty)=k_{B} T / \gamma c$} \\
$\mathrm{Cu}(\mathrm{Mn})$ & $c$ (at.\%) & $T=4.4^{\circ} \mathrm{K}$ & $T=1.6{ }^{\circ} \mathrm{K}$ \\
\hline $2 A$ & $4.30 \times 10^{-2}$ & 3.66 & 1.33 \\
$3 A$ & $8.48 \times 10^{-2}$ & 1.85 & 0.674 \\
$4 A$ & $1.31 \times 10^{-1}$ & 1.2 & 0.436 \\
$5 A$ & $3.43 \times 10^{-1}$ & 0.46 & 0.167 \\
\hline \hline
\end{tabular}

$k_{B} T / \Delta(\infty)=k_{B} T / \gamma c$ for the $\mathrm{Cu}(\mathrm{Mn})$ alloys measured at $T=1.6$ and $4.4^{\circ} \mathrm{K}$.

\section{Initial susceptibility}

Equation (4.6) in the limit $k_{\mathrm{B}} T / \gamma c \ll 1$ gives

$$
\chi_{i}(T) \simeq \frac{2}{\gamma} \frac{N_{0} \mu_{B}^{2}}{\pi} S(S+1)\left[1+\frac{\ln 4}{\pi}\left(\frac{k_{B} T}{\gamma c}\right)\right],
$$

which predicts the qualitative behavior of $\chi_{i} / c$ observed in Fig. 14. A plot of $\chi_{i}(T)$ vs $1 / c$ for $T$ $=4.41 .6^{\circ} \mathrm{K}$ is given in Fig. 15. Clearly the conditions for Eq. (6.2) are not met for $T=4.4^{\circ} \mathrm{K}$ and severe deviations from a straight line are observed. At $T=1.6{ }^{\circ} \mathrm{K}$, however, all samples except $\mathrm{Cu}(\mathrm{Mn})-2 A$ meet the condition, and approximate qualitative agreement with Eq. $(6.2)$ is found, except for the highest-concentration sample $\mathrm{Cu}(\mathrm{Mn})-5 A$, which exhibits remanence at this temperature. This raises a question concerning the range over which this theory may be applicable. At temperatures below $1.6^{\circ} \mathrm{K}$ one would expect samples with con-

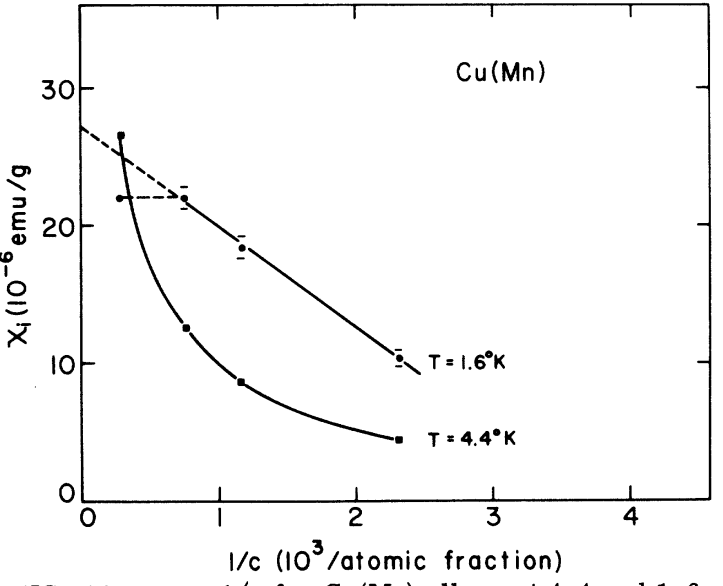

${ }^{\circ} \mathrm{K}$.

centration less than that of $\mathrm{Cu}(\mathrm{Mn})-5 A(0.343$ at. \%) to show remanence and, hence, deviate from the susceptibility predicted by Eq. (6.2). Values for $\gamma$ calculated from the slope and intercept of the fit shown in Fig. 15 give $1.47 \times 10^{3}\left({ }^{\circ} \mathrm{K} /\right.$ at. fraction) and $0.815 \times 10^{3}\left({ }^{\circ} \mathrm{K} /\right.$ at. fraction), respectively. This is an approximate agreement with $\gamma$ as determined previously.

\section{Field-dependent magnetization}

The molecular-field theory discussed in Sec. IV gives a magnetization which depends on applied field, temperature, and impurity concentration. In the MRF approximation given by Klein, ${ }^{15}$ the

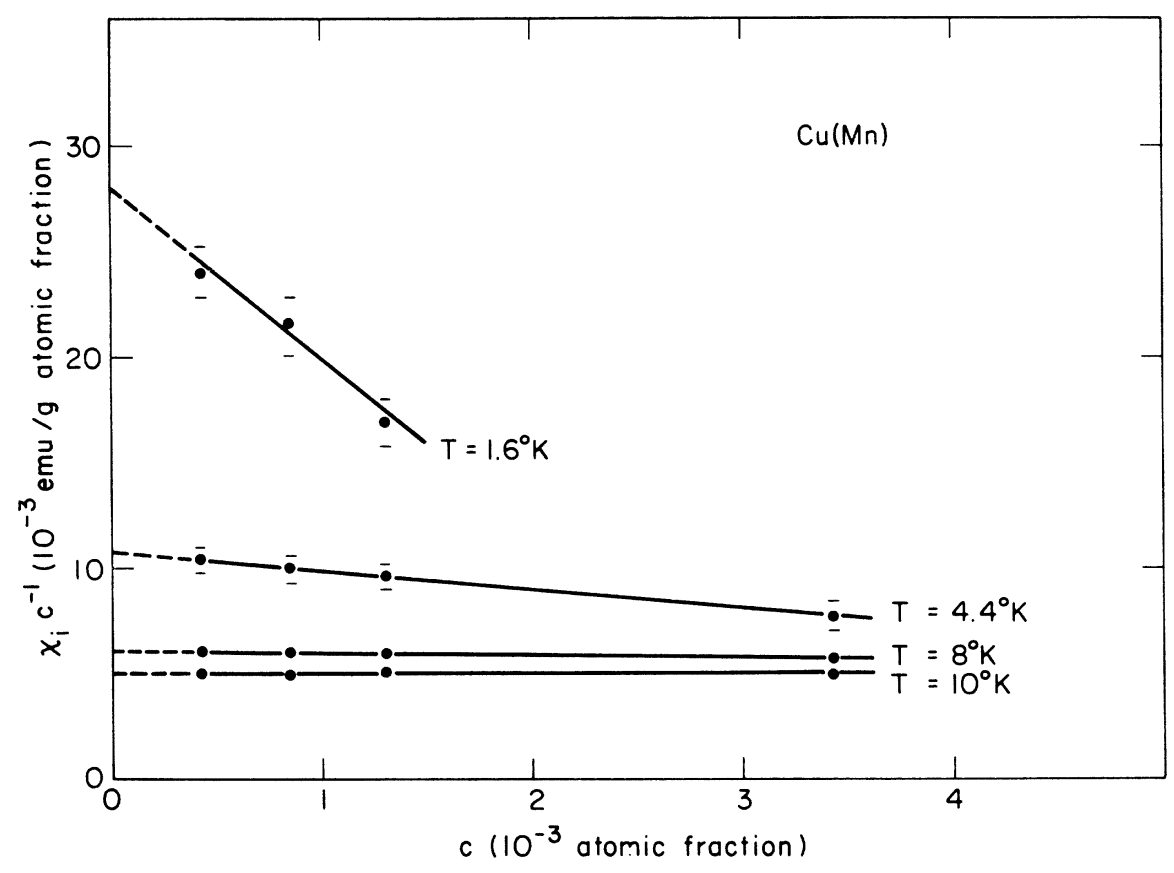

FIG. 14. $\chi_{i} / c$ vs $c$ for $\mathrm{Cu}(\mathrm{Mn})$ alloys as a function of temperature. $\mathrm{Cu}(\mathrm{Mn})-$ $5 A$ shows remanence at $1.6^{\circ} \mathrm{K}$, and is not shown. 


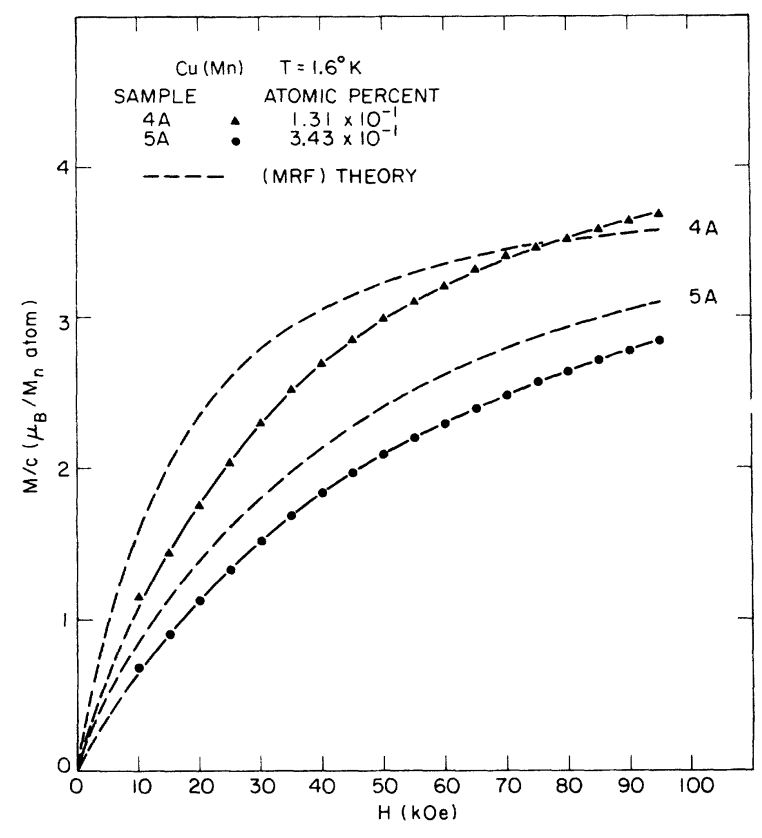

FIG. 16. Comparison of mean-random-field theory with $M / c$ vs $c$ for $\mathrm{Cu}(\mathrm{Mn})-4 A$ and $-5 A$ at $T=1.6^{\circ} \mathrm{K}$.

expression for $M\left(H_{\text {ext }}, c, T\right)$ is given by Eq. (4.8). A comparison of the MRF theory to measured magnetization at $1.6^{\circ} \mathrm{K}$ for $\mathrm{Cu}(\mathrm{Mn})-4 A$ and $\mathrm{Cu}(\mathrm{Mn})-5 A$ is shown in Fig. 16. Here we have taken $\gamma$ $=2.8 \times 10^{3}{ }^{\circ} \mathrm{K} /$ at. fraction, as determined earlier. In order to compare the Ising-model theory to experiment it was necessary to assume that the moment was $q s \mu_{B}=4 \mu_{B}$ rather than simply $\mu_{B}$, as in the original theory. The agreement with experiment is surprisingly good in view of the Ising-model approximation and the approximate value used for $\gamma(\approx \pm 30 \%)$.

\section{SUMMARY AND CONCLUSIONS}

In this work we have attempted to understand the magnetic properties of the two alloy systems $\mathrm{Cu}(\mathrm{Fe})$ and $\mathrm{Cu}(\mathrm{Mn})$ in the finite concentration region, i. e., not in the extreme-dilution limit. We have used our susceptibility and high-field magnetization results, but also have relied heavily on the results of other experiments as explained above. The major results for $\mathrm{Cu}(\mathrm{Fe})$ are as follows:

(i) We have confirmed in part the results of Tholence and Tournier: there exist in $\mathrm{Cu}(\mathrm{Fe})$ alloys isolated iron and pair terms in the magnetization. The pair magnetization saturates at $\sim 60 \mathrm{kOe}$ for $T \simeq 4^{\circ} \mathrm{K}$, and each pair has associated with it a spin of approximately 3 .

(ii) By comparing several completely different sets of samples, it was shown that the pair density depends on sample-preparation procedures such as quench rate and cold work. This, along with Mössbauer evidence ${ }^{44-46}$ for nearest-neighbor $\mathrm{Fe}-\mathrm{Fe}$ interactions, indicates that the magnetic pairs are not due to long-range RKKY-type couplings to distances of $\sim 11 \AA$. Rather, the pairs should be regarded as nearest-neighbor $\mathrm{Fe}$ atoms (in a sense, a diatomic Fe molecule) dissolved in the copper matrix. The pairs form as a result of the great tendency for iron atoms to stick together or cluster in these supersaturated alloys.

(iii) It was shown that the magnetic remanence exhibited by two 0.6 -at. $\%$ alloys was proportional to the pair densities in the respective samples. This lends additional support to the suggestion ${ }^{10}$ that the "ordering" seen in dilute $\mathrm{Cu}(\mathrm{Fe})$ alloys at very low temperatures and concentrations is pairpair ordering.

(iv) It was shown that the resurgence of spinglass transitions in cold-worked alloys, where they did not exist before the working, could be understood on the basis of the breaking up of Fe pairs and clusters with a resulting randomizing effect on the impurity spin position. Thus the magnetic properties of dilute alloys are intimately related to the details of the structure of the alloy.

(v) With respect to the Kondo problem, it was shown that the isolated Fe-atom spin polarization in fields to $\sim 100 \mathrm{kOe}$ and $T \ll T_{K}$ cannot be described by a Brillouin function appropriate to an independent spin. However, a Brillouin function of the form $B_{3 / 2}(\alpha)$, where $\alpha=g \mu_{B} S_{1} H / T+29$, was able to represent the data rather well. So far as we are aware, there is no first-principles theory which is able to describe this result.

For the $\mathrm{Cu}(\mathrm{Mn})$ alloys the present work has shown that the recent mean-random-field theory of Klein is able to explain the concentration dependence of the initial susceptibility in a certain low-temperature limit $(k T \ll \gamma c)$ in which the spins are strongly interacting. In this same limit the theory is able to explain semiquantitatively the field dependence of the magnetization to $\sim 100 \mathrm{kOe}$. The success of this theory in the present work and also in recent high-temperature work makes it apparent that the theory is indeed a good firstorder theory, at least for alloys which approximate well the idealized random solid solution.

\section{ACKNOWLEDGMENTS}

We wish to thank Professor Clifford Shull, Dr. Simon Foner, and Dr. W. M. Star for several very informative discussions concerning experimental techniques and interpretation of the results. Professor B. L. Averbach and Professor R. Kaplow generously provided certain pieces of experimental equipment. We are indebted for 
financial support to the National Science Foundation and the Advanced Research Projects Agency. One of us (J.M. F.) also wishes to acknowledge personal support, in the form of a Resident Graduate Fellowship, from the International Business Machines Corporation.
${ }^{*}$ Research supported at MIT by the National Science Foundation under Grant No. GP-21312, by the Advanced Research Projects Agency under Contract No. DAHC1567-0222, and at the University of Nebraska by the National Science Foundation under Grant No. GH-35690.

†IBM Graduate Fellow. Present address: IBM Corp., Burlington, Vt.

¥Present address (to which correspondence should be sent).

${ }^{1}$ See P. W. Anderson, Phys. Rev. 124, 41 (1961).

${ }^{2}$ J. Kondo, Progr. Theoret. Phys. (Kyoto) 32, 37 (1964).

${ }^{3} \mathrm{~J}$. Kondo, in Solid State Physics, edited by F. Seitz, D. Turnbull, and H. Ehrenreich (Academic, New York, 1969), Vol. 23.

${ }^{4}$ A. J. Heeger, in Ref. 3 .

${ }^{5}$ E. C. Hirschkoff, O. G. Symko, and J. C. Wheatley, J. Low Temp. Phys. 5, 155 (1971).

${ }^{6} \mathrm{~J}$. Owen, M. E. Brown, V. Arp, and A. F. Kip, J. Phys. Chem. Solids 2, 85 (1957).

7J. S. Kouvel, J. Phys. Chem. Solids 21, 57 (1961).

${ }^{8}$ C. M. Hurd, J. Phys. Chem. Solids 30, 539 (1968).

${ }^{9} \mathrm{~J}$. A. Careaga, B. Dreyfus, R. Tournier, and L. Weil, in Proceedings of the Tenth International Conference on Low-Temperature Physics, Moscow, 1966, edited by M. P. Malkov (VINITI, Moscow, 1967).

${ }^{10}$ E. C. Hirschkoff, M. R. Shanabarger, O. G. Symko, and J. C. Wheatley, J. Low Temp. Physics 5, 545 (1971).

${ }^{11}$ C. M. Hurd, J. Phys. Chem. Solids $\underline{28}, 1345$ (1967).

${ }^{12}$ B. Dreyfus, J. Souletie, J. L. Tholence, and R. Tournier, J. Appl. Phys. 39, 846 (1968).

${ }^{13} \mathrm{~J}$. L. Tholence and R. Tournier, Phys. Rev. Lett. 25, 867 (1970).

${ }^{14}$ See also, H. E. Ekstrom and H. P. Myers, Phys. Kondens. Mater. 14, 265 (1972); W. M. Star, F. B. Basters, G. M. Nap, E. de Vroede, and C. van Baarle, Physica 58, 585 (1972).

${ }^{15}$ M. W. Klein, Phys. Rev. 173, 552 (1968); Phys. Rev. 188, 933 (1969).

${ }^{16} \mathrm{~J}$. M. Franz and D. J. Sellmyer, AIP Conf. Proc. $\underline{5}$, 1150 (1972); Bull. Am. Phys. Soc. 17, 293 (1972); J. M. Franz, thesis (Massachusetts Institute of Technology, 1971) (unpublished); D. J. Sellmyer, J. M. Franz, and L. K. Thomas, in Amorphous Magnetism, edited by H. O. Hooper and A. M. deGraaf (Plenum, New York, 1973).

${ }^{17}$ M. Hansen, Constitution on Binary Alloys (McGrawHill, New York, 1958).

${ }^{18}$ R. M. Bozorth, Ferromagnetism (Van Nostrand, New York, 1951).

${ }^{19}$ C. M. Hurd, Cryogenics 6 , 264 (1966).

${ }^{20}$ H. Sato, A. Arrott, and R. Kikuchi, J. Phys. Chem. Solids 10, 19 (1959).

${ }^{21}$ W. Marshall, Phys. Rev. 118, 1520 (1960).

${ }^{22}$ M. W. Klein and R. Brout, Phys. Rev. 132, 2412 (1963).

${ }^{23}$ R. J. Harrison and M. W. Klein, Phys. Rev. 154, 540 (1967).

${ }^{24}$ M. A. Ruderman and C. Kittel, Phys. Rev. 96,99
(1954).

${ }^{25}$ T. Kasuya, Prog. Theoret. Phys. (Kyoto) 16, 45 (1956).

${ }^{26} \mathrm{~K}$. Yosida, Phys. Rev. 106, 893 (1957).

${ }^{27}$ J. E. Zimmerman and F. E. Hoare, J. Phys. Chem. Solids 17, 52 (1960).

${ }^{28} \mathrm{M}$. W. Klein and L. Shen, Phys. Rev. B 5, 1174 (1972).

${ }^{29}$ J. P. Franck, F. D. Manchester, and D. L. Martin, Proc. Roy. Soc. (London) A263, 499 (1961); see also R. J. Potton, D. F. Brewer, and D. J. Emerson, J. Low. Temp. Phys. 9, 135 (1972), and refs. therein.

${ }^{30} \mathrm{~V}$. Jaccarino and L. R. Walker, Phys. Rev. Lett. $\underline{15}$, 258 (1965).

${ }^{31}$ K. Miyatani and S. Iida, J. Phys. Soc. Japan 25, 1008 (1968).

${ }^{32}$ G. R. Caskey and D. J. Sellmyer, J. Appl. Phys. 40 , 1476 (1969).

${ }^{33}$ G. K. Wertheim and J. H. Wernick, Acta Met. $\underline{15}$, 2917 (1967)

${ }^{34}$ H. Claus, A. K. Sinha, and P. A. Beck, Phys. Lett. A 26, 38 (1967).

${ }^{35} \mathrm{~L} . \mathrm{O}$. Creveling, Jr. and H. L. Luo, Phys. Rev. 176, A 614 (1968).

${ }^{36}$ A. Narath and A. C. Gossard, Phys. Rev. 183, 391 (1969).

${ }^{37}$ R. L. Cohen, J. H. Wernick, K. W. West, R. C. Sherwood, and G. Y. Chin, Phys. Rev. 188, 684 (1969).

${ }^{38}$ P. Costa-Ribeiro, J. Soultie, and D. Thoulouze, Phys. Rev. Lett. 24, 900 (1970).

${ }^{39}$ R. Tournier and A. Blandin, Phys. Rev. Lett. 24, 397 (1970).

${ }^{40} \mathrm{~J}$. P. Perrier, B. Tissier, and R. Tournier, Phys. Rev. Lett. 24, 313 (1970).

${ }^{41}$ L. H. Bennett, L. J. Swartzendruber, and R. E. Watson, Phys. Rev. Lett. 23, 1171 (1969).

${ }^{42}$ F. Bitter, A. R. Kaufmann, C. Starr, and S. T. Pan, Phys. Rev. 60, 134 (1941).

${ }^{43}$ L. H. Bennett and L. J. Swartzendruber, Acta Met. $\underline{18}$, 485 (1970).

${ }^{44}$ B. Window, J. Phys. C: Metal Phys. S3, 323 (1970); Philos. Mag. 26, 681 (1972).

${ }^{45}$ S. J. Campbell, P. E. Clark, and P. R. Liddell, J. Phys. F. 2, L114 (1972).

${ }^{46}$ R. C. Knauer, Bull. Am. Phys. Soc. 16, 585 (1971). We are grateful to Dr. Knauer for sending his results prior to publication.

${ }^{47}$ For further discussion of this point, see P. A. Beck, Trans. AIME 2, 2015 (1971); Proceedings of Amorphous Magnetism Conference (Plenum, New York, to be published), and references therein.

${ }^{48} \mathrm{~K}$. Svensson, in Ref. 9, p. 267.

${ }^{49}$ S. B. Nam and J. W. F. Woo, Phys. Rev. Lett. 19, 649 (1967); C. S. Ting, Phys. Rev. B 4, 904 (1971)

${ }^{50}$ P. E. Bloomfield, R. Hecht, and P. R. Sievert, Phys. Rev. B 2,3714 (1970).

${ }^{51}$ A. Luther (private communication).

${ }^{52}$ J. S. Kouvel, J. Appl. Phys. 31, 1425 (1960).

${ }^{53}$ M. W. Klein (private communication). 